3 HongQiang Xia, * Xiyu Fan, Jianli Zhang, Haiming He, and

4 Qingjie Guo

5 State Key Laboratory of High-efficiency Utilization of Coal and Green

6 Chemical Engineering, Ningxia University, Yinchuan 750021, P. R. China 7 8

9

\section{Theoretical Studies on the Oligomerization of Silicate Species in Basic Solution} *Corresponding authors: xhq@nxu.edu.cn

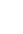

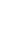

The optimized structures and the relative free energies for all the condensation reactions involved in the reaction pathways.

Figure S1. Optimized structures and relative free energies of the condensation between neutral monomer and anionic monomer to form an anionic dimer $\left(\mathrm{T}_{1}^{-}+\mathrm{T}_{1} \rightarrow \mathrm{T}_{2}^{-}+\mathrm{H}_{2} \mathrm{O}\right)$

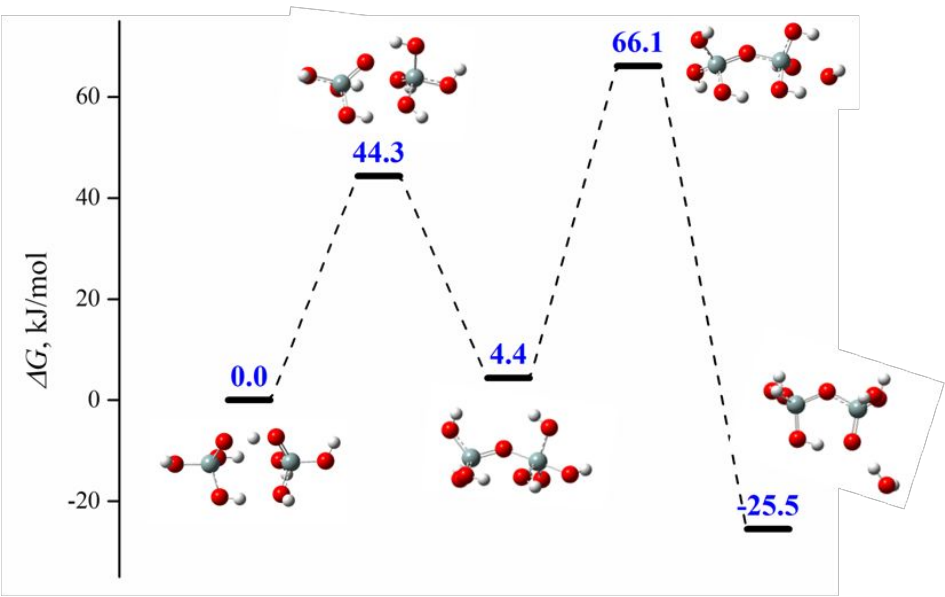


1 Figure S2. Optimized structures and relative free energies of the condensation between

2 neutral monomer and anionic dimer to form an anionic trimer $\left(\mathrm{T}_{2}^{-}+\mathrm{T}_{1} \rightarrow \mathrm{T}_{3}^{-}+\mathrm{H}_{2} \mathrm{O}\right)$

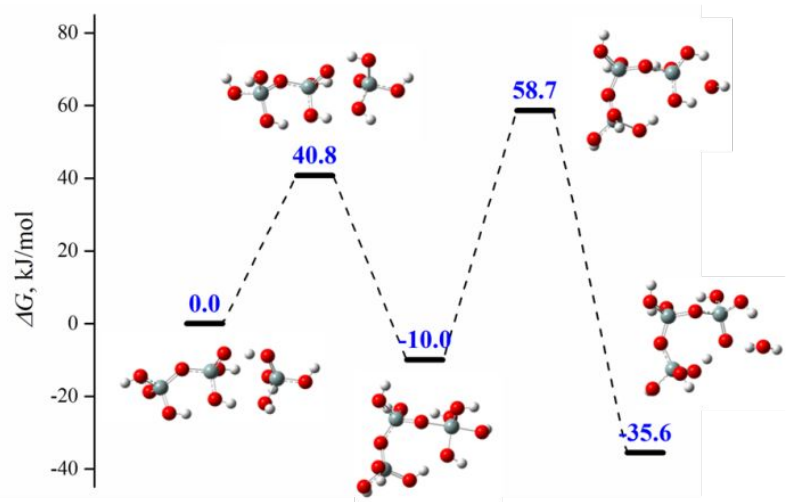

5 Figure S3. Optimized structures and relative free energies of the condensation between 6 neutral monomer and anionic trimer to form an anionic tetramer $\left(\mathrm{T}_{3}^{-}+\mathrm{T}_{1} \rightarrow \mathrm{T}_{4}^{-}+\mathrm{H}_{2} \mathrm{O}\right)$

7

8 Figure S4. Optimized structures and relative free energies of the condensation between neutral monomer and anionic tetramer to form an anionic pentamer $\left(\mathrm{T}_{4}^{-}+\mathrm{T}_{1} \rightarrow \mathrm{T}_{5}^{-}+\mathrm{H}_{2} \mathrm{O}\right)$

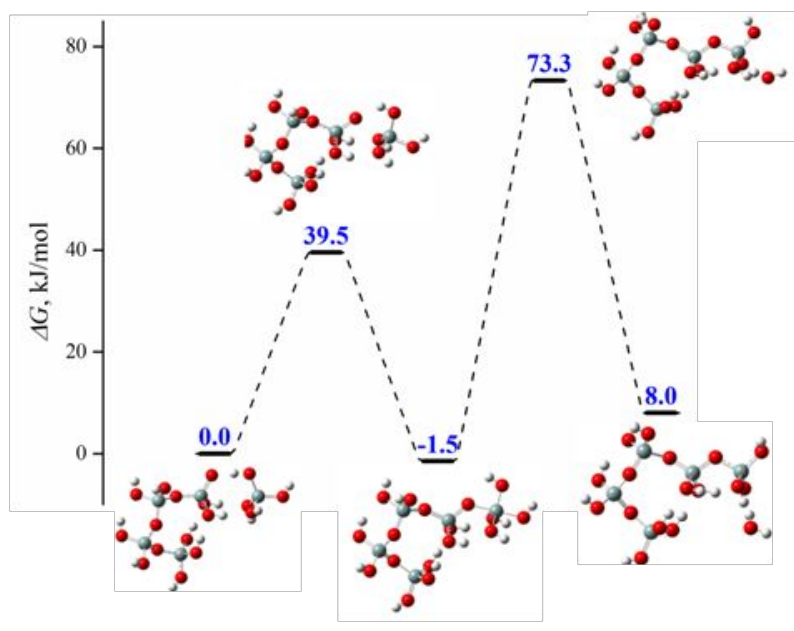


Figure S5. Optimized structures and relative free energies of the condensation

2 between neutral monomer and anionic pentamer to form an anionic hexamer $\left(\mathrm{T}_{5}^{-}+\mathrm{T}_{1}\right.$

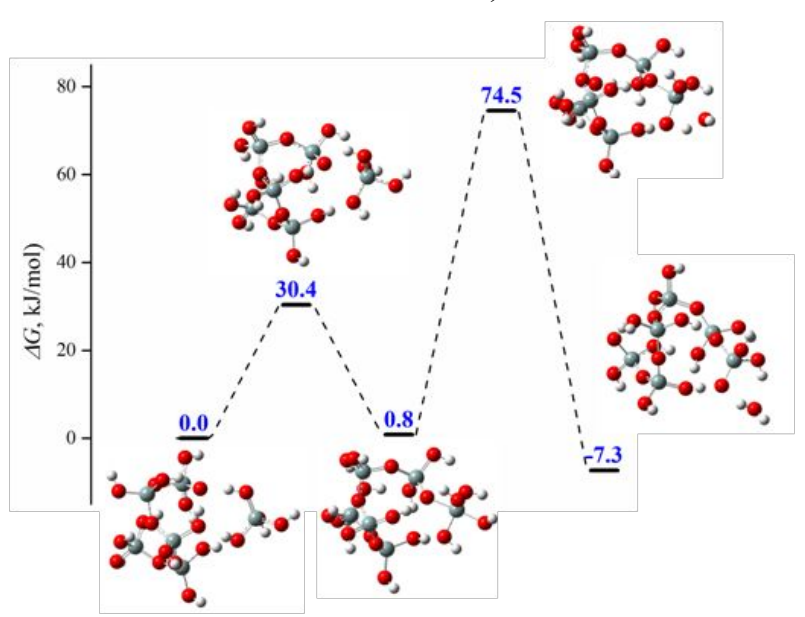

5 Figure S6. Optimized structures and relative free energies of the condensation between 6 a neutral dimer and an anionic dimer to form an anionic tetramer $\left(\mathrm{T}_{2}^{-}+\mathrm{T}_{2} \rightarrow \mathrm{T}_{4}^{\prime}{ }^{-}+\mathrm{H}_{2} \mathrm{O}\right)$

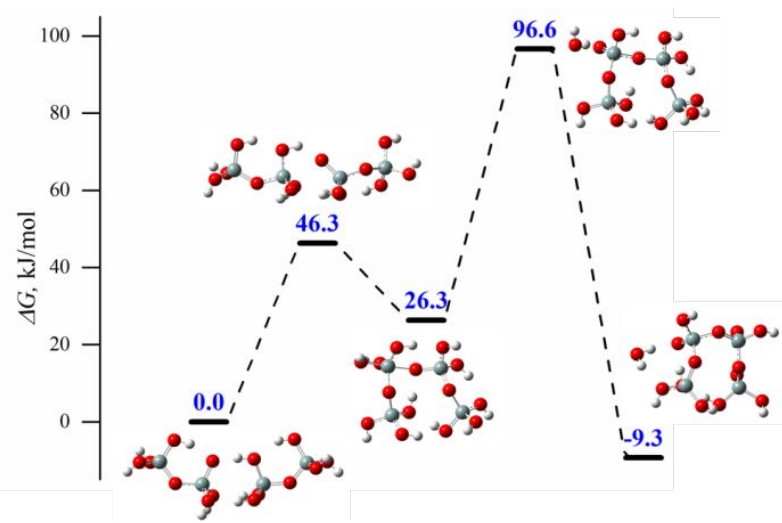

8 Figure S7. Optimized structures and relative free energies of the condensation between

9 a neutral dimer and an anionic trimer to form an anionic pentamer $\left(\mathrm{T}_{3}^{-}+\mathrm{T}_{2} \rightarrow \mathrm{T}_{5}^{\prime}{ }^{-}+\mathrm{H}_{2} \mathrm{O}\right)$

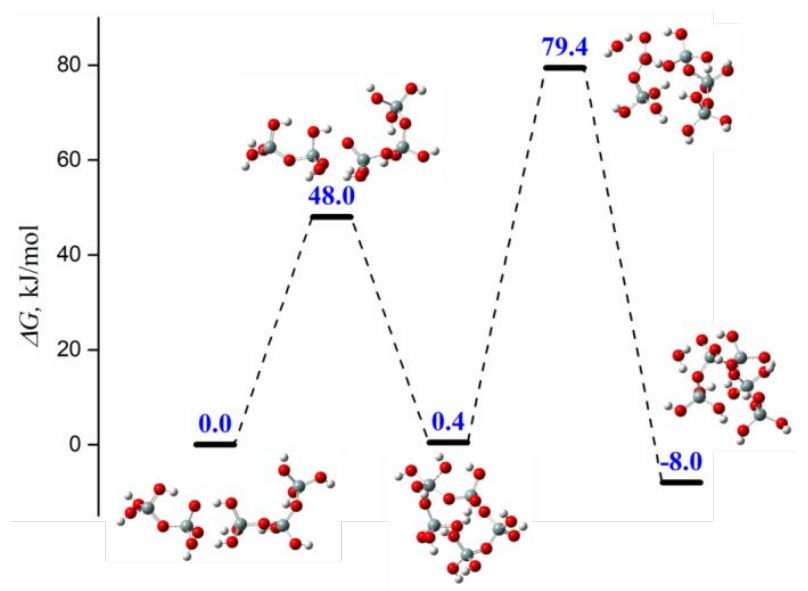


1 Figure S8. Optimized structures and relative free energies of the condensation between

2 a neutral dimer and an anionic tetramer to form an anionic hexamer $\left(\mathrm{T}_{4}^{-}+\mathrm{T}_{2} \rightarrow \mathrm{T}_{6}^{\prime}{ }^{-}+\right.$

$\left.3 \mathrm{H}_{2} \mathrm{O}\right)$

4

5

6 7

8

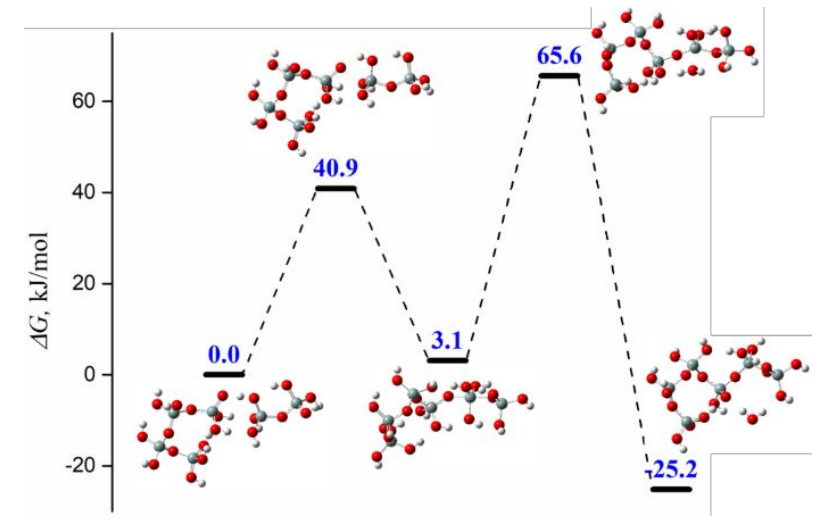

Figure S9. Optimized structures and relative free energies of the condensation between a neutral trimer and an anionic trimer to form an anionic hexamer $\left(\mathrm{T}_{3}^{-}+\mathrm{T}_{3} \rightarrow \mathrm{T}_{6}^{\prime}{ }^{\prime}-\mathrm{H}_{2}\right.$ 0)

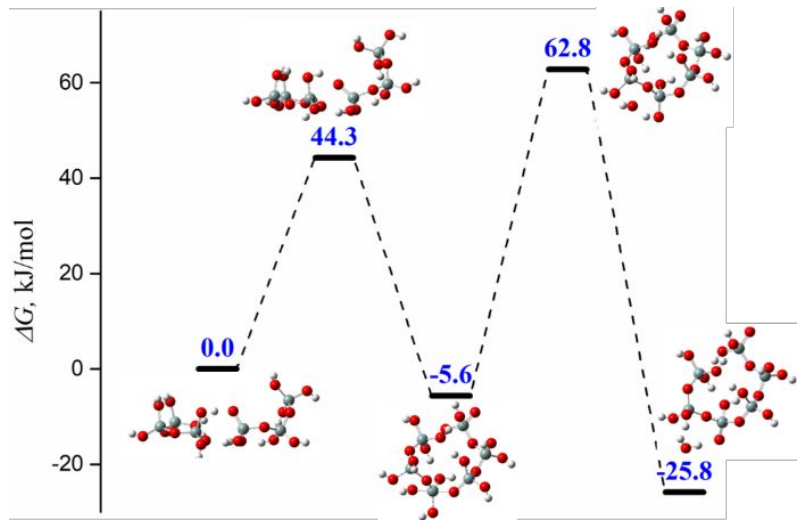

Figure S10. Optimized structures and relative free energies of the cyclization reaction of an anionic trimer to 3-ring $\left(\mathrm{T}_{3}^{-} \rightarrow \mathrm{R}_{3}^{-}+\mathrm{H}_{2} \mathrm{O}\right)$

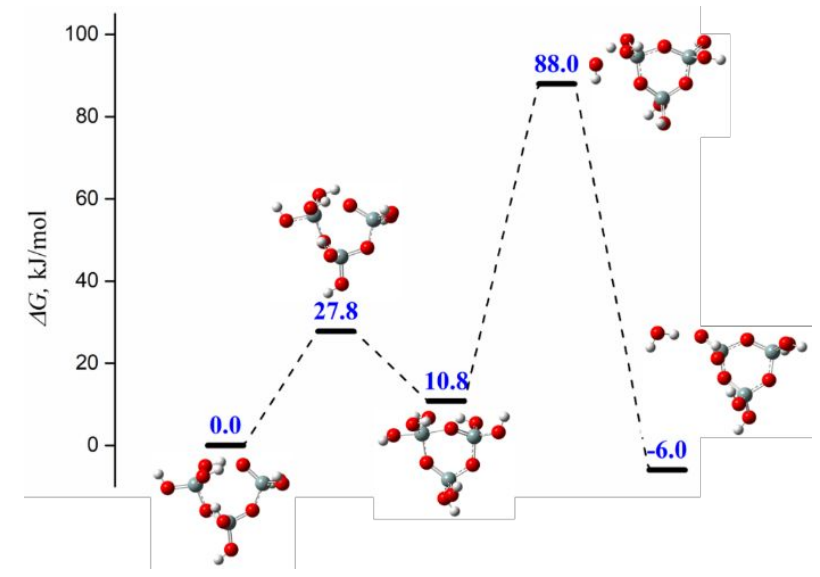


1 Figure S11. Optimized structures and relative free energies of the cyclization reaction 2 of an anionic tetramer to 4-ring $\left(\mathrm{T}_{4}^{-} \rightarrow \mathrm{R}_{4}^{-}+\mathrm{H}_{2} \mathrm{O}\right)$

3

4 Figure S12. Optimized structures and relative free energies of the cyclization reaction 5 of an anionic pentamer to 5-ring $\left(\mathrm{T}_{5}^{-} \rightarrow \mathrm{R}_{5}^{-}+\mathrm{H}_{2} \mathrm{O}\right)$

7

8

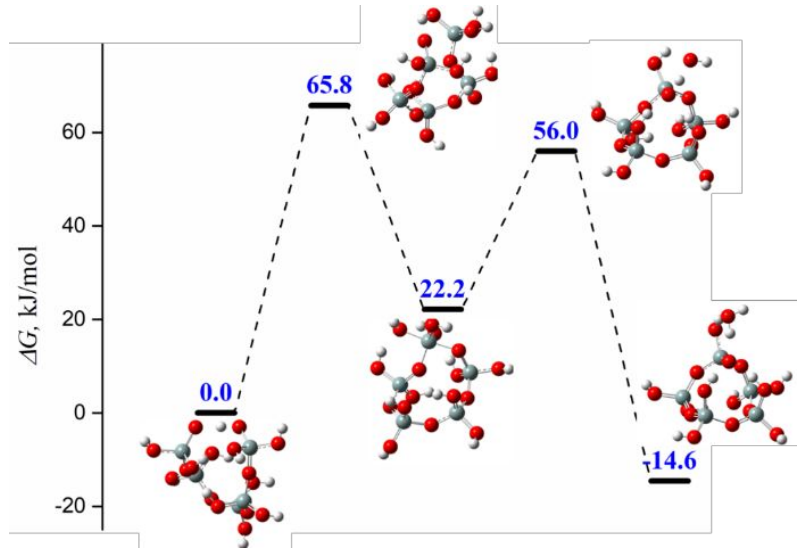

Figure S13. Optimized structures and relative free energies of the cyclization reaction of an anionic hexamer to 6-ring $\left(\mathrm{T}_{6}^{-} \rightarrow \mathrm{R}_{6}^{-}+\mathrm{H}_{2} \mathrm{O}\right)$
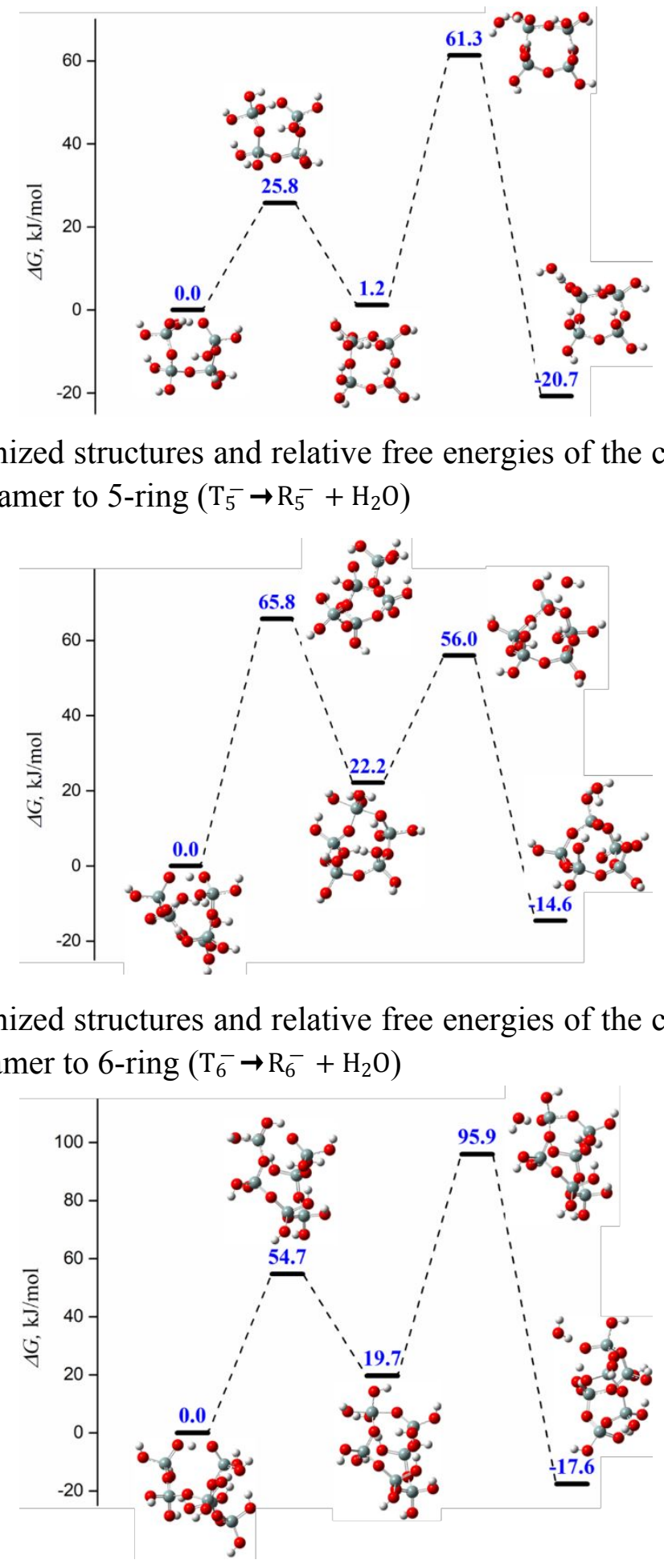
1 Figure S14. Optimized structures and relative free energies of the cyclization reaction 2 of an anionic tetramer to a branched 3-ring $\left(\mathrm{T}_{4}^{-} \rightarrow \mathrm{R}_{3}^{1-}+\mathrm{H}_{2} \mathrm{O}\right)$

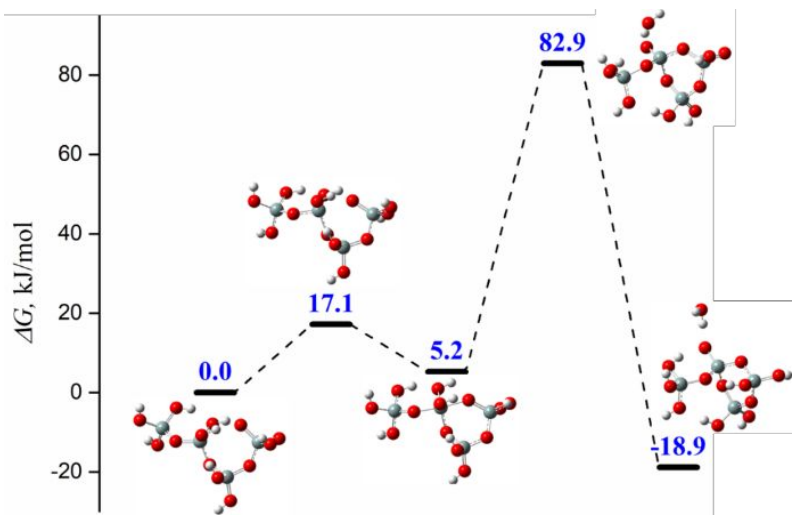

4 Figure S15. Optimized structures and relative free energies of the cyclization reaction 5 of an anionic pentamer to a branched 3-ring $\left(\mathrm{T}_{5}^{-} \rightarrow \mathrm{R}_{3}^{2-}+\mathrm{H}_{2} \mathrm{O}\right)$

6

7 Figure S16. Optimized structures and relative free energies of the cyclization reaction

8 of an anionic pentamer to a branched 4-ring $\left(\mathrm{T}_{5}^{-} \rightarrow \mathrm{R}_{4}^{1-}+\mathrm{H}_{2} \mathrm{O}\right)$

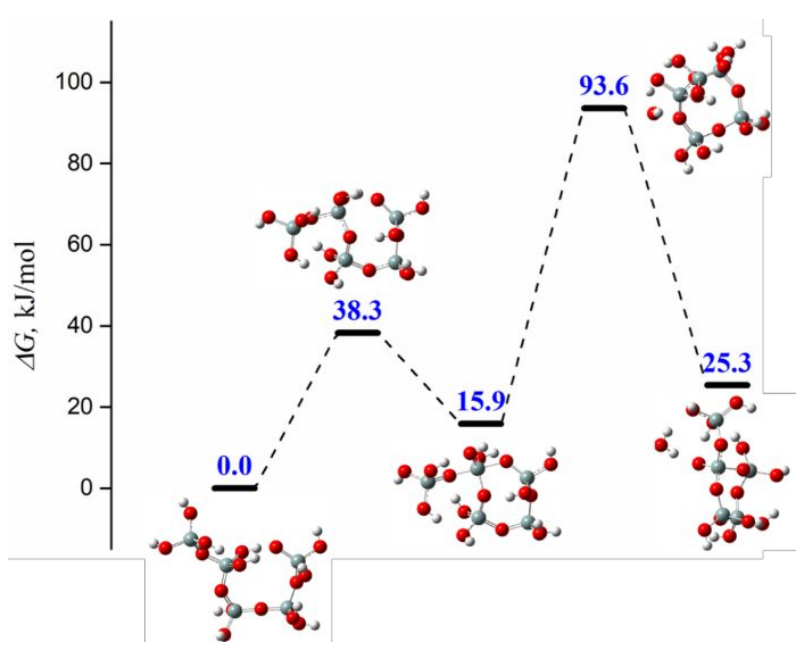


1 Figure S17. Optimized structures and relative free energies of the cyclization reaction

2 of an anionic hexamer to a branched 3-ring $\left(\mathrm{T}_{6}^{-} \rightarrow \mathrm{R}_{3}^{3-}+\mathrm{H}_{2} \mathrm{O}\right)$

3

4 Figure S18. Optimized structures and relative free energies of the cyclization reaction

5 of an interior charged hexamer to a branched 4-ring $\left(\mathrm{T}_{6}^{\prime}{ }^{-} \rightarrow \mathrm{R}_{4}^{2-}+\mathrm{H}_{2} \mathrm{O}\right)$

6

7 Figure S19. Optimized structures and relative free energies of the cyclization reaction

8 of an anionic hexamer to a branched 5-ring $\left(\mathrm{T}_{6}^{-} \rightarrow \mathrm{R}_{5}^{1-}+\mathrm{H}_{2} \mathrm{O}\right)$

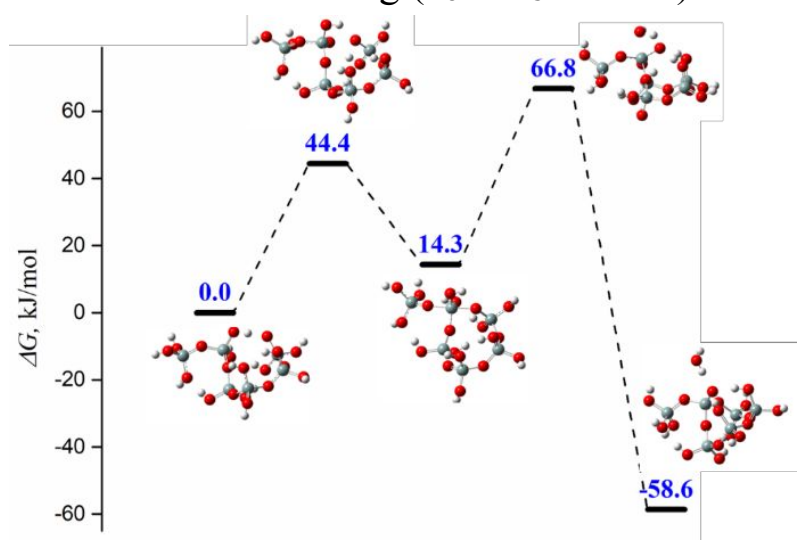


1 The optimized geometries of all the reactants, products, TSs and

2 intermediates involved in the reaction pathways

$$
\mathbf{T}_{1}^{-}+\mathbf{T}_{1} \rightarrow \mathbf{T}_{2}^{-}+\mathrm{H}_{2} \mathbf{O}
$$

Reactant

O - $1.19009500-0.177116001 .49045100$

Si - $1.90946400-0.009756000 .07781600$

O 1.271529000 .028916001 .53620400

Si 1.904544000 .011535000 .05578900

O $3.55408200-0.011986000 .08879800$

O $-1.620211001 .46431300-0.66265800$

O $-1.48581600-1.21782900-1.00900500$

O $1.306149001 .31903200-0.78666100$

O $1.44410700-1.29102300-0.87717800$

O $-3.57785700-0.027361000 .11666400$

H $0.20905300-0.057671001 .55960600$

H -3.95533700 -0.66297400 0.72977100

H 3.979621000 .499822000 .78253000

H - $0.53254300-1.39637900-1.02868800$

H $-0.669426001 .62713900-0.76234000$

H $1.81192700-2.14501900-0.62940300$

H $1.610470001 .41459700-1.69486700$

TS1

O -1.82841500 -0.19639600 1.69595700

Si - $1.70798800-0.001178000 .06750600$ O $0.61211400-0.066039001 .11403900$

Si $1.80199900-0.013149000 .06401800$

O $3.37046000-0.075728000 .64401200$

O - $1.110669001 .44551600-0.51226700$

O -1.06996100 -1.30670800 -0.76265000

O $1.735471001 .40115700-0.84211000$

O $1.79347800-1.33331500-0.96797400$

O -3.29795800 $0.09073600-0.45520100$

H -0.88702500 -0.22799500 1.97507200

H - $1.787189001 .97379100-0.94812900$

H - $1.67377500-1.63469000-1.43702800$

H $0.810859001 .69654000-0.85822300$

H $0.86871800-1.60965300-1.06748800$

H -3.94079200 -0.38633700 0.07605500

H 3.656900000 .715136001 .10796000

Intermediate

Si $1.727211000 .01238300-0.00987500$

Si -1.424834000 .089449000 .05488500$
O 0.262524000 .656335000 .18741200

O $2.815198001 .19324800-0.44763200$

O $2.40229100-0.667722001 .34557600$

O $1.68289900-1.20827500-1.12346000$

O -1.93223100 $1.70834500-0.08731200$

O -0.98647600 -0.83959500 -1.32345600

O - $1.29689100-0.672517001 .56716700$

O -3.11405200 -0.43994300 -0.12047300

H $2.480040001 .84431900-1.07031400$

H $2.65605000-0.051894002 .03832700$

H $0.75359400-1.31055800-1.41430500$

H - $1.170388002 .29341400-0.04221200$

H -3.69996700 $0.30994400-0.24673500$

H -1.76605900 -1.29275000 - 1.65833700

H -2.15263900 -1.05713100 1.78084400

TS2

Si - $1.84632900-0.057206000 .00166000$

O - $1.59858600-1.44989700-0.84481300$

O -2.84475400 $0.97711100-0.80519400$

O -0.40432700 0.637578000 .32879600

Si 1.215635000 .263756000 .09918300

O $1.792284001 .58977900-0.72025500$

O 1.747840000 .167734001 .66555600

H $2.69024500-0.045304001 .61600800$

O $1.12022200-1.12817200-0.80043500$

H -2.53964700 $1.27859500-1.66516500$

H $1.100724002 .23241100-0.90399400$

O $3.47718200-0.61589900-0.30194900$

H $3.93718900-1.247160000 .26115600$

H $2.10731700-1.31889600-0.85752100$

O -2.72354300 -0.33064900 1.37036900

H -2.34780000 - 0.954115001 .99776800

H - $0.64886800-1.61792800-0.99665400$

Product

O -2.99511600 -0.05660100-1.08145300

Si - $1.794868000 .30579500-0.00212400$

O -2.56371500 0.469853001 .45010500

O -0.68028900 - 0.902585000 .01316400

O - $1.039271001 .72157300-0.32858000$

Si $0.99695200-0.764983000 .00068900$ 
O $1.57110300-1.309563001 .46617800$

O $1.53695000-1.92162100-1.07696600$

O $1.402652000 .74467900-0.28156800$

H -3.19706200 -0.21516400 1.68147000

H -2.71096000 -0.32569400 - 1.95958600 H $2.858594001 .50460700-0.16767600$

H -0.05085800 $1.55775900-0.35324800$ H 1.44159300 -2.24554900 1.64240700 H $1.23869800-1.80800200-1.98361800$ O $3.725635001 .99149100-0.11814700$ H 3.927222002 .062854000 .81847100

$\mathbf{T}_{2}^{-}+\mathbf{T}_{1} \rightarrow \mathbf{T}_{3}^{-}+\mathrm{H}_{2} \mathbf{O}$

Reactant

O -3.02338800 - $1.15497800-1.43685000$

Si -3.06942000 -0.27452200 -0.04763200

O $-0.69841800-0.30665500-0.96347400$

Si $0.306852000 .59975800-0.14801400$

O $1.913513000 .56070200-0.60025200$

O $-2.26502200-0.869378001 .29041400$

O -2.81519000 $1.36864700-0.23134600$

O 0.377589000 .125586001 .47594900

O -0.06899900 $2.22481600-0.21656500$

O $-4.65957300-0.402785000 .46339900$

$\mathrm{H}-2.08588700-1.08575200-1.71371900$

H -2.86811500 -1.18170900 1.97275900

$\mathrm{H}-3.555898001 .886528000 .10087300$

H $-0.44956200-0.336449001 .69481000$

H - $1.035008002 .29087400-0.28071800$

Si $3.21273100-0.25232100-0.04087200$

O $3.03807200-0.586981001 .56054000$

H 2.10748200 -0.47336600 1.83609000

O $3.33283600-1.60965300-0.97998700$

H $4.06809500-2.20158600-0.79776200$

O $4.597979000 .63962000-0.14389600$

H $4.991683000 .72129900-1.01706400$

H -5.31027000 -0.55218600 -0.22747100 TS1

O $2.96135900-0.88925000-1.19014400$ Si $3.362329000 .13884900-0.01296400$ O $0.48301300-1.19526000-1.30311700$ Si $-0.37656000-0.55062300-0.13903800$ O -2.02100900 - $0.76610100-0.27911800$ O $2.592666001 .61522800-0.09777800$
O $2.83168900-0.497421001 .43117900$

O -0.25459200 $1.13251100-0.06087100$

O -0.02288600 - 1.152059001 .38025900

O $4.978037000 .46471000-0.01167300$

H $1.93173200-1.05446400-1.27426300$

Si -3.33424700 0.16644500-0.01799300

O -2.92568300 $1.75905300-0.02114300$

O $-4.38710100-0.21461500-1.23153600$

H -5.29350100 $0.08962900-1.13254500$

O $-4.03611300-0.111230001 .45423900$

H -4.44358200 -0.97293400 1.58087300

H $5.56937000-0.26762900-0.20816700$

H $0.654078001 .47377600-0.09358800$

H $0.92974100-1.120004001 .55995600$

H $2.887188002 .21161400-0.79340900$

H 3.006115000 .028753002 .21829400

H - $1.957498001 .88133300-0.03959600$ Intermediate

Si -2.38938000 -0.76050600-0.02838900 Si -0.379562001 .519700000 .01411600$ Si $2.19316600-0.429353000 .07961300$ O -3.83696400 -0.93016800-0.80222100 O -1.37800300 - 1.91491100-0.58205800 O -2.69108800 -0.85834500 1.59290200 O - $1.810392000 .75475500-0.27044200$ O 0.778694000 .551816000 .52773900 O -0.790122002.69636600 1.10359300 O $0.164319002 .19134200-1.40944900$ O 2.923627000 .043010001 .53668600 O $1.17092000-1.815717000 .10576600$ O $2.447309000 .40563000-1.37602300$ O $3.58492700-1.47622600-0.29943700$ H -3.80074000 -1.07407600 -1.75203600 H $-0.42255000-1.86330900-0.31617500$ H -2.95206200 -1.72036200 1.92905400 $\mathrm{H}-0.063565003 .236351001 .42740600$ H -0.43429100 $2.74677900-1.91561800$ H $1.70109000-2.58047400-0.14239900$ H $1.795104001 .09262000-1.56663100$ H $3.93849200-1.26897100-1.16760600$ H $3.79358700-0.366758001 .57279000$ TS2

O - $1.23966800-2.01429000-0.61898900$ Si -2.29615200 -0.92828200-0.01248900 
O -2.53541100 -1.06038000 1.61381700 O - $1.797934000 .61911800-0.24442400$ O $-3.75182900-1.15228000-0.74989300$ Si -0.519145001 .601719000 .00583700$ O $0.059299002 .16630200-1.44290900$ O -1.09203300 2.80372800 0.96920400 O 0.741866000 .837942000 .67949500 Si $2.01519800-0.131055000 .15149400$ O $2.408262000 .47867800-1.34133200$ O 3.120475000 .187324001 .34179300 H $3.89342400-0.360494001 .14858900$ O $1.29140800-1.628660000 .11944000$ H - $0.563763002 .52069700-2.08341600$ H -0.47665100 3.506489001 .19753000 H -3.74076700 -1.26168000 - 1.70499800 H -0.28902200 - $1.91470100-0.36549000$ H -2.72672900 -1.93969100 1.95224600 H $1.769130001 .13669700-1.65312800$ O $3.67441500-1.83673200-0.40276600$ H $4.19077200-2.333479000 .24064500$ H $2.11420200-2.15318200-0.14726500$ Product

Si -2.06598100 - $1.07609000-0.08981900$ Si -0.66050100 1.631962000 .29299200 Si $1.962147000 .21293300-0.17206700$ O -3.59445500 - $1.32925100-0.66295400$ O $-0.99284100-1.66581700-1.15169300$ O - $1.99598500-1.756287001 .41516000$ O -1.865861000 .536605000 .19300700$ O 0.758501000 .968712000 .72957500 O - 1.195561002 .722010001 .40280200 O -0.35243900 2.32456800 - 1.18290600 O 3.262388000 .011362000 .84026500 O $1.45464100-1.17383700-0.77959100$ O $2.457952001 .36048900-1.27679400$ O $2.94877300-2.852927000 .64008500$ H -3.72505800 -1.17476700 - 1.60278500 H $0.00547000-1.46973100-1.00337500$ H -1.99905200 -2.71705200 1.44677300 H - 0.630634003 .482523001 .56715200 H - $1.085818002 .71235400-1.66848200$ H $1.721494001 .88122400-1.62390900$ H $3.57314500-3.330884000 .08708800$ H $3.42892700-0.931736001 .00557600$
H $2.33132100-2.380210000 .02083300$

$$
\mathbf{T}_{3}^{-}+\mathbf{T}_{\mathbf{1}} \rightarrow \mathbf{T}_{\mathbf{4}}^{-}+\mathbf{H}_{2} \mathbf{O}
$$

Reactant

O -3.48005300 - $1.37938300-1.12060100$

Si -4.22456200-0.67519700 0.12659500

O - $1.17547300-0.44237200-1.39524600$

Si $-0.799512000 .83072200-0.53340100$ O $0.754420001 .40120400-0.74064700$

O -3.37423200 -0.74072900 1.55937100

O $-4.335356000 .95460300-0.19128100$

O -0.834516000 .549990001 .13360300$

O - $1.739939002 .17364200-0.84315100$

O -5.68786700 -1.36923400 0.43311300

H -3.33274600 -1.60006800 1.99106100

$\mathrm{H}-4.752478001 .495067000 .48739400$

$\mathrm{H}-1.614533000 .060293001 .44325900$

H -2.68379300 $1.95648900-0.79450800$ Si 2.126598001 .350333000 .12577400

O 1.800217000 .856905001 .67525100 H 0.841310000 .697366001 .80222100 O $3.219504000 .28188900-0.48609400$ O 2.789024002 .851527000 .05704600 H 3.729153002 .922512000 .24456900 H $-6.20613000-1.64770700-0.32726600$ Si $3.64867900-1.25504900-0.09516900$ O $5.22162100-1.56165800-0.46303900$ H $5.88351500-1.223032000 .14679900$ O $2.73321000-2.22741400-1.05458900$ H $2.89054900-3.17447100-1.00625500$ O $3.41278100-1.492073001 .51791800$ H $2.81509300-0.832438001 .90926900$ H -2.51350600 -1.02052700 -1.28295500 TS1

O -3.32906200 - $1.97786500-0.99255900$ Si -3.78553100 -0.78795900 0.04859500 O - $1.45652900-0.29343600-0.82255700$ Si $-0.886522001 .09641300-0.33689900$ O $0.690238001 .46424200-0.75208800$ O -2.97272300 -0.65066100 1.50219900 O -4.05317500 $0.71117900-0.64293200$ O -0.83403700 1.176382001 .35551000 O - $1.743440002 .40659600-0.91032300$ O $-5.29438200-1.278931000 .58670600$ 
H -3.51368700 -0.91996000 2.25170000 H -4.95071100 $1.02365100-0.48806800$ H - 1.493035000 .552284001 .70684300 H -2.66897600 $2.12750100-0.99460700$ Si 2.116042001 .361028000 .01935600 O 1.861709001 .013306001 .62142000 H 0.903624001 .047320001 .83446900 O $3.070841000 .15842900-0.57055600$ O $2.885793002 .79244200-0.22335900$ H $3.833282002 .81222500-0.06229500$ H -5.79148400 -1.84027900 -0.01394700 Si $3.37391600-1.38996500-0.11153700$ O $4.91901800-1.83445500-0.45728400$ H $5.60183700-1.535547000 .15029000$ O $2.39039800-2.32862000-1.03602700$ H $2.49205100-3.28186500-0.96523200$ O $3.11031100-1.536848001 .50729500$ H $2.61978700-0.782466001 .87734100$ H -2.44305300 -1.69021000 - 1.29327400 Intermediate

Si -2.03611500 - 1.857136000 .16744300 O -3.15581000 -2.72952700 1.00421000 O -2.22731100 -1.94684700 - 1.47446400 O -2.31558500 -0.30095100 0.63528800 Si -2.22903000 $1.12729000-0.16209000$ O -0.69661600 $1.39699400-0.68404600$ O -3.24137200 $0.94972400-1.44757900$ O -2.72122500 2.34896200 0.81679600 Si 0.685605001 .747794000 .15406100 O 0.075638002 .572789001 .46781400 O $1.631180002 .71984000-0.80329000$ O 1.577041000 .504298000 .58144000 Si $2.83372000-0.62199000-0.02702400$ O $3.615484000 .56772400-0.93510500$ O $3.22784600-0.899349001 .60498100$ H $4.00558500-1.459438001 .67337600$ O $1.55059900-1.51827000-0.76110900$ O -0.55912600 -2.40695500 0.56187100 H - 1.987955002 .718239001 .33343400 H 0.654717002 .679510002 .22766500 H -3.27318700 $1.65252600-2.10293800$ H -2.80504200 -1.27700100 -1.86158200 H -3.04388300 -3.68411100 1.00944200 H $0.22338300-2.040789000 .07321700$
O $4.04364900-1.77501700-0.62082400$ H 3.94259400 -2.66492100 -0.27206200 H $1.91230200-2.21712900-1.31524300$ H $3.157980001 .41721000-0.93755100$ H $1.219846003 .46517800-1.24909200$ TS2

Si -1.97939200 $1.93493800-0.14804300$ O -3.15361000 $2.78412300-0.93157000$ O -2.13405500 1.966196001 .49831500 O -2.19979600 $0.38274500-0.66339100$ Si -2.23711500 - 1.060059000 .10378600 O $-0.74058600-1.434116000 .67560000$ O -3.27736500 - 0.844918001 .35878800 O $-2.75100600-2.23785600-0.91475300$ Si $0.63619600-1.93037100-0.06501700$ O $0.08236800-2.93974200-1.25108100$ O $1.62329800-2.662203001 .03649700$ O $1.50607000-0.72010300-0.68421000$ Si $2.639452000 .39190800-0.12157900$ O $3.53892200-0.456362000 .98462400$ O $3.418661000 .71308700-1.54680200$ H $4.086577001 .38442500-1.35493300$ O 1.665952001 .563155000 .54526200 O $-0.535654002 .55721200-0.55814000$ H -2.02978000 -2.73564400 -1.32759400 H $0.69924500-3.23604300-1.92661900$ H -3.40253000 - 1.569358001 .97866000 H -2.73576100 1.309279001 .87122300 H -3.09633900 $3.74264200-0.88855100$ H $0.279351002 .18222000-0.13928100$ O 3.997019002 .280249000 .61901700 H 4.223546003 .043428000 .07723700 H 2.419653002 .207812000 .77292100 H $3.14797800-1.313380001 .20749600$ H $1.25835600-3.336951001 .61612500$ Product

Si - 1.592137002 .109988000 .03284700 O -2.71437300 $3.16022100-0.57614800$ O - 1.740190001 .909573001 .67281800 O - $1.977921000 .67303100-0.69363300$ Si -2.31833200 -0.81287500 -0.11040100 O - $0.96536800-1.501237000 .52725800$ O $-3.42781100-0.569486001 .07921000$ O $-2.91220000-1.77655800-1.29828100$ 
Si $0.39358200-2.10086600-0.16988000$

O -0.17608600 -2.85933200-1.52245600

O $1.15872300-3.097958000 .89742000$

O $1.46637400-0.95301400-0.55697600$

Si 2.512912000 .034005000 .31435500

O $2.66665700-0.702729001 .80269300$

O $3.97635800-0.08534400-0.47409100$

H $3.612923003 .04776900-1.83303900$

O 2.027099001 .545220000 .41822700

O -0.11241600 $2.64457700-0.31875600$

H - $2.23861800-2.32772100-1.72304800$

H $0.45322900-3.16065400-2.18407500$

H -3.71942000 -1.32952600 1.59116700

H -2.47580300 1.350744001 .95324100

H -2.55161500 $4.09388300-0.41584000$

H 0.728649002 .153752000 .00447100

O $3.949559002 .75460900-0.98157600$

H $3.157494002 .46713300-0.45552400$

H $2.44876100-1.643213001 .82215800$

H $0.64138500-3.782441001 .33209900$

H $4.251434000 .77415100-0.83417100$

$\mathbf{T}_{4}^{-}+\mathbf{T}_{1} \rightarrow \mathbf{T}_{\mathbf{5}}^{-}+\mathrm{H}_{2} \mathbf{O}$

Reactant

O -4.63831200 - $1.29210700-0.24578100$

Si -4.62508100 0.23106400 0.29196600

O - $2.26330700-2.08324100-0.55719200$

Si - $1.12152100-0.99723700-0.50326600$

O $0.42772800-1.50883800-0.80155000$

O -3.68985400 0.463674001 .65144900

O -3.88115700 $1.17493700-0.86126100$

O - $0.98377600-0.226183000 .99128100$

O - $1.288566000 .22584600-1.65033900$

O -6.13635100 0.758735000 .67195800

H -4.03403000 0.108446002 .47726300

H -3.81320900 $2.11521000-0.66471800$

H - $1.84735000-0.006327001 .38467200$

H -2.17942400 $0.61636700-1.61018000$

Si $1.70998300-2.10960800-0.01208100$

O $2.85148300-2.60884000-1.10332200$

H $2.57510800-3.17308400-1.83112300$

O $2.49953000-0.964418000 .85763700$

O $1.17177200-3.299886000 .98100700$

H $1.81858000-3.734143001 .54473200$
Si 3.579335000 .202299000 .41866900

O 4.258609000 .883501001 .74733700

H 4.807163000 .319262002 .29968200

O $2.814440001 .39629700-0.38287700$

O $4.73917400-0.46996600-0.53335200$

H $4.43773200-1.26042200-1.00584900$

H -3.69119200 - $1.67642000-0.39739200$

Si $1.456603002 .33459600-0.29099700$

O $1.935348003 .90999900-0.23745900$

H 2.739291004 .095803000 .25498300

O $0.583312002 .19288200-1.66402500$

H $-0.095462001 .48237400-1.69503300$

O 0.611663001 .972954001 .06435100

H 0.053140001 .163701001 .06824300

H -6.85470000 0.490862000 .09176300

TS1

O -5.10584300 - $1.36234700-0.12077700$

Si -4.52765600 0.15099700 0.16916000

O -2.58670600 -1.36348600-0.36078300

Si - $1.19547100-0.62561400-0.37254100$

O $0.16722700-1.50448400-0.72590900$

O -3.59940200 0.401859001 .53644300

O -3.89340800 $0.95952500-1.15422800$

O -0.842324000 .073334001 .12120300$

O - $1.140792000 .59668500-1.52885800$

O -5.899882001 .028139000 .55843700$

H -4.06422200 0.916520002 .20453300

H -4.37613900 $1.77170100-1.34116900$

H - 1.690191000 .287413001 .55139900

H -2.05231900 $0.92447500-1.63799600$

Si $1.43103500-2.209597000 .00264500$

O $2.41907400-2.89091800-1.13998200$

H $2.02087600-3.44379200-1.81856200$

O $2.41468100-1.132506000 .75197800$

O $0.84178200-3.287186001 .09166800$

H $1.47965000-3.777111001 .61883500$

Si $3.58077400-0.103794000 .20429800$

O 4.500575000 .432384001 .45253900

H $5.02390300-0.220985001 .92558200$

O $2.901844001 .21435600-0.47190100$

O $4.50851000-0.89152100-0.90151500$

H $4.07595300-1.66939500-1.28555800$

H -4.31138300 -1.88643700 -0.33882300

Si $1.773957002 .37228900-0.12146500$ 
O 2.532174003 .829126000 .00351600 H 3.380969003 .835004000 .45396200 O $0.740964002 .53764000-1.37547800$ H $0.043027001 .84993500-1.46742200$ O 1.036470002 .016264001 .29827100 H 0.362661001 .299095001 .28660300 H -6.72928100 0.678698000 .22226800 Intermediate

O -5.12415000 - $1.32909600-0.11035300$ Si -4.19379500 0.070877000 .10117600 O - $2.71697200-0.88794300-0.27606800$ Si - $1.21912400-0.31080800-0.31318900$ O - $0.07622000-1.42254300-0.69806800$ O -3.486602000.484780001.61023900 O -3.79327100 $1.11908400-1.19787000$ O -0.80251000 0.308486001 .17902200 O - $1.086784000 .87416300-1.47723200$ O -5.63793300 1.004089000 .46652000 H -4.08275900 1.059623002 .10006100 H $-4.453235001 .81569900-1.26729900$ H - 1.638538000 .517924001 .64729200 H - $1.987535001 .23087400-1.62606300$ Si $1.14258800-2.26840800-0.02960600$ O $2.02472600-2.99299000-1.22530400$ H $1.56305300-3.50650100-1.89462000$ O $2.22854700-1.299813000 .72026100$ O $0.47111500-3.315613001 .03615600$ H 1.06294500 -3.89164400 1.52892800 Si $3.46974400-0.361237000 .17381700$ O 4.441056000 .080861001 .41829800 H $4.90673200-0.618168001 .88623700$ O $2.885330001 .01326200-0.47612700$ O $4.31623800-1.20407200-0.95517900$ H $3.81403000-1.92455400-1.36407600$ $\mathrm{H}-4.56346000-2.07668200-0.33762500$ Si $1.991171002 .34700400-0.08708500$ O 2.973415003 .662520000 .01119900 H 3.782409003 .563770000 .52027900 O $0.963133002 .68868200-1.31054300$ H $0.206747002 .07654100-1.42912800$ O 1.250190002 .109755001 .35780600 H 0.514939001 .461072001 .37563600 H -6.42797200 0.459141000 .44673300 TS2
O -5.29576900 - $1.32533200-0.05449700$ Si -4.03679300 -0.26290500 0.00954400 O -2.64648700 - $1.17895400-0.24996800$ Si -1.17606800 -0.47605900 -0.26894900 O $0.02762200-1.51672600-0.58728700$ O -3.70376500 0.408845001 .49255400 O -3.99868700 $0.79302500-1.26800100$ O -0.875006000 .214182001 .21145000$ O - $1.143749000 .67396000-1.46541100$ O -5.60388600 1.678757000 .44715500 H -4.42619900 1.093251001 .53718400 H -4.68575800 $1.45040800-0.97012600$ $\mathrm{H}-1.707386000 .484285001 .64321200$ H -2.04100000 $1.00144100-1.66417800$ Si $1.33063700-2.273543000 .04127700$ O $2.20445500-2.95278300-1.18242500$ H $1.76023300-3.53266900-1.80803100$ O $2.36695400-1.212053000 .72665400$ O $0.75737800-3.338019001 .14293500$ H $1.39364100-3.880534001 .61821800$ Si $3.51966300-0.196619000 .12184800$ O 4.514522000 .314849001 .31966300 H $5.05455700-0.346735001 .76142700$ O $2.814372001 .13291500-0.49992100$ O $4.36564600-0.98628700-1.04394100$ H $3.90308400-1.74741300-1.42428200$ H -5.10218700 -2.21453400 0.25731900 Si $1.846495002 .40412200-0.08644000$ O 2.736272003 .781962000 .00561900 H 3.546137003 .744482000 .52159700 O $0.776376002 .68245500-1.29094300$ H $0.082700002 .00837500-1.42515300$ O 1.146904002 .113110001 .37043500 H 0.448431001 .431145001 .40306800 H -6.54654000 1.507302000 .51525000 Product

O $-5.32762600-1.49043700-0.13215300$ Si -4.01717800 -0.48172300-0.05780200 O -2.63898000 -1.46214100-0.13497200 Si - $1.20058900-0.69219400-0.21453100$ O $0.04603500-1.67229400-0.57439500$ O -3.87028200 0.183289001 .48085200 O $-3.968866000 .63268300-1.19099800$ O -0.87894800 -0.01247500 1.27283500 
O - $1.296597000 .46994500-1.38726800$ O -4.21559200 2.780609000 .37405800 H -4.08289500 1.133754001 .46057800 H -4.16167300 $2.10727800-0.35452800$ H - 1.703998000 .204698001 .73895100 H -2.26158600 $0.70706400-1.50614500$ Si $1.42332000-2.294453000 .04383300$ O $2.34661400-2.90617600-1.17896400$ H $1.96435300-3.55336700-1.77877300$ O $2.36180900-1.130366000 .70305900$ O $0.96765300-3.394829001 .16621900$ H 1.66121500 -3.86901900 1.63431900 Si $3.44674800-0.044382000 .09558300$ O 4.402252000 .535396001 .29466800 H $4.98044700-0.089422001 .74177500$ O $2.661002001 .23547500-0.53491500$ O $4.34671400-0.78252500-1.06362200$ H $3.94227200-1.58164100-1.43233300$ H -5.41767800 -2.12904100 0.58094800 Si $1.583289002 .41620800-0.11916500$ O $2.345898003 .87086300-0.06136100$ H 3.185818003 .904636000 .40430600 O $0.465760002 .57828300-1.30012700$ H -0.19148400 $1.85729100-1.38834600$ O 0.946162002 .080884001 .35705500 H 0.321142001 .332333001 .41270100 H -5.07823200 3.197567000 .29594800

$\mathbf{T}_{5}^{-}+\mathbf{T}_{1} \rightarrow \mathbf{T}_{6}^{-}+\mathrm{H}_{2} \mathbf{O}$

Reactant

O $3.896840001 .12244800-0.45169300$

Si $4.13477000-0.47493000-0.27094500$ O $1.390009001 .81390600-0.40070800$ Si $0.293515001 .72696700-1.56741000$ O - $1.176093002 .23757300-0.95381000$ O $3.00418200-1.180303000 .72027900$ O $4.02227300-1.19808300-1.75322100$ O $0.068293000 .18913100-2.17255200$ O $0.516219002 .71416100-2.87502200$ O $5.59619900-0.777048000 .43301100$ H $2.50753100-0.584326001 .31871200$ H $4.22557200-2.13655800-1.80186700$ H $0.42060800-0.54551400-1.63039100$ H $1.184156002 .43659300-3.50784500$
Si -1.45291000 2.480951000 .64214800 O -0.31519900 3.480137001 .28559200 H 0.561252003 .295321000 .90906000 O -1.449519001.00798500 1.40679500 O -2.956732003.06043100 0.91610200 H -3.21907100 3.858654000 .44874600 H $6.38100800-0.52448200-0.06143800$ Si -0.330103000 .078105002 .18920900$ O -0.628631000 .230707003 .79145000$ H -0.15353700 -0.34753900 4.39498600 O - $0.58904500-1.480581001 .74538600$ O 1.184911000 .548708001 .79142700 H 1.266899001 .064392000 .91180800 H $2.948061001 .43761100-0.51317200$ Si $-0.50132500-2.438658000 .41480400$ O $0.74301600-1.95438400-0.55270700$ H $1.61365000-1.79050700-0.12493900$ O -0.39403200 -4.00632000 0.88115300 H $0.29833200-4.241233001 .50558800$ O $-1.84908200-2.32660500-0.47873500$ Si -3.07326400 - $1.38585400-1.05034800$ O $-3.63080600-0.411462000 .14756700$ H -2.96497600 0.050146000 .68031800 O -2.56461900 -0.60868800 -2.39845700 H - $1.63998000-0.28440200-2.39414600$ O $-4.37788700-2.30387800-1.43900900$ H -4.34141800 -2.76297600 -2.28260200 TS1

O 4.396982000 .915301000 .79603500 Si $4.10499700-0.43711300-0.09220100$ O 1.900423000 .820176000 .20015600 Si $1.101615001 .37975200-1.06230800$ O -0.08823600 2.46635800 -0.65087900 O $3.13109900-1.609093000 .59605600$ O $3.68969900-0.11932200-1.67427100$ O $0.304015000 .14493500-1.88030300$ O $2.024126002 .22421600-2.15081100$ O $5.55966400-1.25063500-0.13620100$ H $3.63005800-2.386014000 .87172600$ H $4.21468900-0.61012700-2.31453900$ H $0.53793300-0.73029800-1.51022800$ H $2.837321001 .72873000-2.32216300$ Si - 1.518162002 .471481000 .13208200 O -1.65227100 3.803138001 .08263600 
H -0.866460004 .070581001 .56751500$

O - 1.685764001 .073315001 .01168100

O $-2.791028002 .55843300-0.89391500$

H -2.84427600 $1.78375600-1.48322100$

H $6.35165500-0.71076700-0.20399200$

Si -0.843903000 .225927002 .15966100$

O - 1.757651000 .328318003 .52101700

H - $1.43303700-0.126954004 .30302300$

O -0.80587200 - 1.335546001 .64990700

O 0.643668000 .817792002 .40063400

H 1.212525000 .813002001 .55124400

H 3.546328001 .391907000 .82894300

Si - $0.68365000-2.378817000 .40094100$

O $0.71469800-2.15887200-0.43390400$

H $1.55279600-1.941929000 .03690300$

O -0.86584200 -3.91460000 0.95247300

H - $0.22213700-4.234527001 .59093100$

O - $1.88293800-2.15685300-0.68122800$

Si -3.06236800 - $1.14126500-1.19929800$

O -3.92545100 -0.50515300 0.04351000

H -3.42261500 0.136339000 .56740900

O -2.37279500 $0.05436400-2.10310200$

H - $1.384786000 .08405200-2.11284500$

O -4.18684900 - $1.97201300-2.05733100$

H -3.88683400 -2.42134400 -2.85226200

Intermediate

O 4.393865000 .626196000 .97859000

Si $3.68729300-0.47844600-0.08831200$

O $2.131385000 .46775400-0.03119200$

Si $1.263873001 .19653000-1.19376500$

O $0.276523002 .32434000-0.51655400$

O $2.77982000-1.811019000 .49810000$

O $3.72104900-0.22211500-1.77353400$

O $0.292014000 .08025100-1.96199400$

O $2.174842002 .01925900-2.28758100$

O $5.14209000-1.45195800-0.11588100$

H $3.37164100-2.558971000 .63383200$

H $4.50197500-0.63802900-2.15164400$

H $0.39962100-0.82627700-1.60003100$

H $2.956858001 .47899200-2.48943400$

Si - 1.139019002 .531507000 .27202800

O -1.052071003.84158700 1.25226600

H - 0.243461003 .958481001 .75907700

O -1.49329600 1.147685001 .11730600
O $-2.386685002 .83518600-0.73632000$

H -2.57722400 $2.09173300-1.33840200$

H $5.87173900-1.023645000 .33788200$

Si -0.712744000 .137739002 .16817600$

O -1.49199400 0.304272003 .59937200

H - $1.24854400-0.294203004 .31130800$

O $-0.90103200-1.385961001 .60121300$

O 0.857755000 .535584002 .33149500

H 1.400843000 .465123001 .50951400

H 3.809849001 .365061001 .17088700

Si - $0.94466600-2.373253000 .29670700$

O $0.44506100-2.25859100-0.56688100$

H $1.31784600-2.13612300-0.09689500$

O - $1.29006000-3.902607000 .77827700$

H $-0.64501700-4.350385001 .33308900$

O -2.14998900 - $1.94753500-0.71550900$

Si -3.21634900 -0.77986900-1.14853100

O $-3.95004900-0.086921000 .14547300$

$\mathrm{H}-3.354718000 .454212000 .68451400$

O -2.41198000 0.35990900-2.03415600

H - $1.437736000 .24751600-2.11773700$

O $-4.46343000-1.42959000-1.99008400$

H $-4.25163300-1.87819700-2.81342700$

TS2

O 4.435636000 .563190001 .08197800

Si $3.52131700-0.24822200-0.05188600$

O 2.089864000 .723226000 .01608300

Si $1.166347001 .32767800-1.19375000$

O $0.086071002 .37477600-0.55107600$

O $2.90769500-1.723574000 .36032000$

O $3.856078000 .09911500-1.66090100$

O $0.335428000 .09599300-1.93298800$

O $2.042535002 .18534300-2.28368600$

O $5.11481800-1.65631500-0.18742800$

H $4.05515600-2.100928000 .18052500$

H $4.62854500-0.38434500-1.97168600$

H $0.50308800-0.80007900-1.55568800$

H $2.874847001 .72443700-2.47337800$

Si - 1.359021002 .499156000 .20865200

O -1.387712003.84496000 1.13909000

H -0.61485200 4.021828001 .68299500

O -1.61436600 1.120620001 .09495700

O -2.60121600 2.66703600-0.83556600

H -2.71767700 $1.89027500-1.41437400$ 
H $5.75171000-1.580398000 .53051900$ Si - 0.752108000 .196705002 .16214300 O - 1.608507000 .231601003 .55686200 H - $1.36726900-0.396290004 .24382700$ O $-0.67738800-1.319018001 .56777600$ O 0.738362000 .818315002 .40649800 H 1.330731000 .780606001 .62771800 H 4.027964001 .381887001 .37957900 Si -0.70089900 -2.38296900 0.32143900 O $0.64114100-2.22943700-0.59598300$ H $1.56100900-2.08594400-0.15944300$ O -0.93033400 -3.89899700 0.90953200 H -0.20892000 -4.29174900 1.40912100 O - $1.98156500-2.09039700-0.64760000$ Si -3.12398200 -1.02287000 - 1.13351800 O -3.93308900 -0.34759900 0.12385400 H -3.39373400 0.257979000 .65316700 O -2.39866600 $0.15428700-2.04092100$ H - $1.420902000 .11259100-2.12418800$ O $-4.30178300-1.79232700-1.97407300$ H $-4.03686700-2.26895200-2.76562600$ Product

O -4.04848400 -0.70736900 1.77005200 Si -3.37074700 -0.05901400 0.40601700 O - $1.93430100-0.914060000 .17482000$ Si - $1.16368300-1.26835900-1.23830400$ O $0.02066400-2.34903600-0.92981200$ O -3.13041100 1.507709000 .54537100 O -4.26366200 -0.46920200 -0.94998100 O - $0.479941000 .10871100-1.84902600$ O -2.20000100 - $1.96064300-2.30097000$ O -4.68774200 2.24977000 - 1.53897200 H -4.12593600 2.21170300 -0.72821300 H $-4.635804000 .32526000-1.37741400$ H - $0.688999000 .93748500-1.34838700$ H -3.10066400 - $1.61437800-2.18139100$ Si $1.53181300-2.42888000-0.29175700$ O $1.75857500-3.887855000 .41309400$ H $1.04648500-4.229603000 .96154800$ O $1.71401400-1.173071000 .77245400$ O $2.69610700-2.30717000-1.42670000$ H $2.71893100-1.43136600-1.85705000$ H -5.50255100 2.69302600 -1.28550000 Si $0.92552100-0.544108002 .08216300$
O $2.01209100-0.625184003 .30460200$ H $1.81453900-0.141424004 .11152300$ O 0.515222000 .997113001 .74822300 O -0.39701700 -1.43361800 2.45337300 H - $1.10909200-1.361841001 .79289300$ H -4.26767600 -1.64314600 1.73825400 Si 0.455620002 .263257000 .70387200 O $-0.881669002 .19300600-0.22208500$ H - 1.826128001 .944657000 .17074500 O 0.604579003 .664751001 .54922400 H - 0.126893003 .913441002 .12135000 O $1.744580002 .22358200-0.29715100$ Si $2.929164001 .40491100-1.06744800$ O $3.915150000 .59510500-0.03526100$ H $3.48522700-0.144956000 .41527500$ O $2.240223000 .34887400-2.14023900$ H $1.260130000 .30706200-2.15376600$ O $3.949819002 .44425500-1.82001900$ H $3.568467003 .03941200-2.47142300$

$\mathbf{T}_{\mathbf{2}}^{-}+\mathbf{T}_{\mathbf{2}} \rightarrow \mathbf{T}_{\mathbf{4}}^{\prime-}+\mathbf{H}_{\mathbf{2}} \mathbf{O}$

Reactant

O - $1.314254000 .84240000-0.02300600$ Si - $1.86342500-0.707409000 .05761700$ O $1.244247000 .72554000-0.08475000$ Si $1.90677700-0.71303400-0.05655500$ O $3.56725600-0.66246500-0.10077600$ O $-1.45427600-1.57448200-1.28227700$ O - $1.37558400-1.466946001 .43526900$ O $1.38410900-1.63283600-1.35965700$ O $1.45241600-1.539449001 .33373000$ O $-3.50176100-0.624060000 .10679000$ H $-0.302084000 .90621200-0.05267400$ Si $4.526904000 .67176000-0.00153300$ O $3.606391002 .02075600-0.13805500$ H $2.643732001 .78637800-0.11989600$ O 5.297901000 .529603001 .45363000 H 5.967127001 .188203001 .66029600 O $5.626899000 .73645500-1.22976400$ H $6.384156000 .14797100-1.16248100$ Si -4.57615600 0.61229800-0.00373000 O -3.80381600 2.054572000 .13670500 H -2.83486800 1.970909000 .06576200 O -5.67397400 0.586979001 .22350200 
H -6.37594600 -0.06727000 1.16510600 O -5.31658100 $0.39505200-1.46262600$ H -6.02659900 $1.00325100-1.68748600$ H - $0.49680800-1.67647500-1.43673300$ H - $0.41056300-1.568566001 .53450500$ H $1.85544800-2.400351001 .48359600$ H 1.76720900 -2.50983900 -1.46089300 TS1

O - $1.72368400-0.66882000-1.30093000$ Si - 1.664340000 .248000000 .06920800 O $0.68452100-0.11501800-0.93362300$ Si $1.810589000 .66519400-0.14636200$ O $3.388377000 .42436200-0.62956300$ O -0.91631700 -0.45127700 1.38208100 O - $1.214530001 .83644200-0.12883500$ O 1.852727000 .197201001 .47980700 O $1.638781002 .32348200-0.22207000$ O -3.26702500 0.342023000 .51588400 $\mathrm{H}-0.78023100-0.72021300-1.57895800$ H - $1.50908900-0.577499002 .13033400$ H - 1.910857002 .457559000 .10863700 H $0.98190800-0.159174001 .72233800$ H $0.690640002 .52230000-0.25729300$ Si $4.62345900-0.47732300-0.06070400$ O $4.41729100-0.794895001 .54020400$ H $3.50686300-0.579883001 .82271400$ O $4.65235300-1.84181900-0.99602000$ H $5.35546900-2.47398500-0.82192600$ O $6.069100000 .31383500-0.16072300$ H $6.467486000 .37086600-1.03376100$ Si $-4.66565800-0.32682400-0.00824200$ O -4.49217300 -0.91972200 - 1.53146300 H -3.55887100 -1.02802700 - 1.78225600 O $-5.876909000 .78537500-0.12097300$ H -6.29210500 1.056605000 .70266700 O -5.03282700 - 1.483123001 .11288000 H -5.84539000 -1.97957400 0.97964400 Intermediate

Si - 1.156086001 .989017000 .02405200 O -0.18143000 $2.90865700-0.93948400$ O -2.42120100 2.94690200 0.48076900 O - $1.758228000 .69011200-0.72417300$ Si -2.33188800 -0.80974000-0.13781600 O - $0.67393700-1.151037000 .62132100$
O -2.88442000 -0.81383600 1.46677700

O - $2.16485000-2.08910200-1.22415800$ Si $0.76732600-1.709996000 .20017500$ O $0.49989100-2.97824100-0.83802000$ O $1.62607200-2.199338001 .52358100$ O $1.69468000-0.59110300-0.60275900$ H $2.49435400-1.779640001 .61382700$ Si $2.892778000 .45210300-0.22315300$ O $3.75720200-0.340169000 .94342700$ O $3.882529000 .75387100-1.49204200$ H $3.585650001 .38974200-2.14932900$ O 2.271560001 .885928000 .29662600 H 1.510210001 .794519000 .90665300 O -0.248379001 .504623001 .34235300$ H - $1.30651000-2.53426900-1.22766200$ H 1.25063700 -3.34303100 -1.31448300 H 4.436175000 .142213001 .42361900 H -0.35393000 0.538133001 .45577000 H - 2.199944003 .817966000 .82208100 H $0.761498002 .69726300-0.88733900$ O -3.98180700 -0.54977900 -0.67645500 H -4.09265900 -0.76795700 -1.60473200 H -3.83673700 -0.67420100 1.45386900 TS2

Si - 1.202229002 .003921000 .05197400 O -0.28308400 $2.88454100-0.98736400$ O -2.51747900 2.901862000 .45569600 O - $1.754548000 .62216400-0.59736000$ Si - $2.05304100-0.90631600-0.01059700$ O $-0.55797800-1.258042000 .73056200$ O -3.06433700 -0.99577500 1.26737800 O -2.15642300 - $2.00538500-1.24211000$ Si $0.88696000-1.757632000 .18387000$ O $0.52704700-2.93619200-0.91818900$ O $1.80997800-2.328745001 .41441200$ O $1.69619500-0.53800100-0.57297100$ H $2.61725800-1.816302001 .57484900$ Si $2.892635000 .52406100-0.22527000$ O $3.72018700-0.219958000 .99606100$ O $3.901983000 .76017100-1.48994000$ H $3.638991001 .39310400-2.16446200$ O 2.255630001 .979960000 .20042500 H 1.536085001 .930135000 .85987600 O -0.233821001 .668997001 .37257000$ 
H - $1.34600100-2.52625300-1.35412800$ H 1.24055100 -3.31040800 -1.44288000 H 4.438632000 .248171001 .43077100 $\mathrm{H}-0.324101000 .769848001 .71742100$ H -2.35940900 3.804812000 .74562600 H $0.666508002 .69782000-0.96438000$ O $-4.43633400-0.44617000-0.62975200$ H -4.78331100 -1.20838000 - 1.10300200 H -3.92187900 -0.79441500 0.70529100 Product

Si $1.80806600-1.37913900-0.59812500$ O $1.03611300-2.46136400-1.56473600$ O $3.43183200-1.68739300-0.66789800$ O $1.634438000 .17396900-1.07707500$ Si $1.415406001 .48132000-0.03492400$ O -0.07808800 $2.09682400-0.43668200$ O 1.591982000 .996271001 .46600700 O $2.502510002 .65729800-0.47005300$ Si - $1.630054001 .64831300-0.28262400$ O - $2.404424002 .46283500-1.48426300$ O -2.25555300 2.048303001 .18722900 O - $1.773813000 .01835900-0.48973400$ H -2.57615200 1.289693001 .69941000 Si -2.40181300 -1.28579100 0.27456700 O -2.92681300 -0.66618600 1.71592400 O -3.69120300 - $1.92106900-0.51538100$ H -3.51476900 -2.57689100 - 1.19604100 O - $1.26754000-2.458789000 .41582400$ H - $0.41205000-2.168232000 .80739600$ O $1.20282900-1.536368000 .93977000$ H $2.358379003 .06637300-1.32804300$ H -3.36536700 2.44185400 - 1.46588700 H $-3.37270300-1.245475002 .34016700$ H $1.26704500-0.633327001 .37962400$ H $3.70473800-2.60381600-0.56162000$ H $0.11826200-2.64168300-1.31981200$ O 4.199596000 .101673001 .72213400 H 4.20082500 -0.51710700 0.97974300 H 3.294406000 .487869001 .71201200 $\mathbf{T}_{3}^{-}+\mathbf{T}_{\mathbf{2}} \rightarrow \mathbf{T}_{\mathbf{5}}^{\prime-}+\mathbf{H}_{\mathbf{2}} \mathbf{O}$

Reactant

O $2.439913000 .53829700-0.73923100$ Si $3.05790200-0.547368000 .23280800$ O - $0.064527000 .28610400-1.36342100$
Si $-0.59655500-1.01341600-0.53667700$ O -2.20316500 -1.21256700 -0.81484500 O $2.23789400-0.555443001 .70069900$ O $2.89701800-2.08500300-0.42184500$ O -0.48286700 -0.79393100 1.10859400 O $0.14451600-2.40854600-0.99539800$ O $4.66830300-0.297956000 .55459800$ H $2.56916500-1.143828002 .38664500$ H $3.28711300-2.815844000 .06798500$ H $0.41679700-0.680577001 .47303900$ H $1.11475200-2.42038000-0.89361200$ Si -3.56297300 -1.13526400 0.08418200 O -3.18711500 -0.70768400 1.63878100 H -2.22772600 -0.71320800 1.81657900 O -4.59556200 $0.00731800-0.47722900$ O $-4.27403600-2.60627200-0.03944800$ H -5.19071800 -2.68068000 0.24060800 Si -4.93032900 $1.56012800-0.05035700$ O $-6.486559001 .95862900-0.39102000$ H -7.16078200 1.645952000 .21919800 O -3.97211600 2.49236100 -1.00491300 H $-4.067890003 .44635400-0.93512300$ O -4.660639001.75047500 1.56187500 H -4.084382001.064343001.93669200 H $0.912175000 .46775200-1.19209800$ Si 5.616729000 .932983000 .01049800 O $4.751832001 .88118600-1.00771800$ H $3.811042001 .57084300-1.05846700$ O $6.911205000 .38328600-0.85284800$ H $7.63015200-0.01322400-0.35267900$ O 6.127303001 .709553001 .37563700 H 6.792287002 .395158001 .26672200 TS1

O $2.236509000 .85590700-0.97057400$ Si $2.53327800-0.47870900-0.04895600$ O $0.06605500-0.37337400-0.81267100$ Si -0.77716200 - $1.60583000-0.30001900$ O -2.40763500 - $1.61991000-0.66729500$ O $1.78711800-0.506176001 .43914900$ O $2.42619400-1.94731200-0.82014100$ O -0.80377000 - 1.670428001 .39183600 O $-0.24080800-3.06906100-0.88891400$ O $4.15283100-0.330789000 .30556000$ H $2.40993700-0.532266002 .17302600$ 
H $3.26477900-2.41920300-0.85311000$ H $0.01105100-1.257197001 .72392700$ H $0.71484000-2.99775600-1.03854400$ Si -3.70688900 -0.99822100 0.08634700 O -3.34057000 -0.71161000 1.67853600 H -2.43737500 -1.02985300 1.89194000 O -4.18078400 $0.44319100-0.54987700$ O -4.92838200 - 2.07579700-0.11571300 H -5.82408300 - 1.754261000 .01970900 Si -3.90801800 $2.01149300-0.14214800$ O -5.16509300 $2.97846400-0.57287400$ H -5.93876900 $2.96368300-0.00194000$ O -2.61286800 $2.48216200-1.03746500$ H -2.34304800 $3.40305200-0.98179900$ O -3.67206700 2.115512001.48376800 H -3.47061800 1.255458001 .89032600 H $1.279782000 .77391100-1.18920300$ Si 5.296408000 .823392000 .10832800 O $4.806707001 .94070600-0.99256000$ H $3.856801001 .87821500-1.19075800$ O $6.697235000 .20833400-0.50200300$ H $7.26126300-0.273550000 .10935000$ O 5.538751001 .446954001 .61733000 H 6.213414002 .125842001 .70859400 Intermediate Si $2.53758200-1.046597000 .99280300$ O 2.880438000 .425678000 .35639400 O $3.14045600-0.980829002 .51104600$ O $3.25779900-2.259064000 .14795100$ H $2.74836300-2.53714800-0.62793700$ Si $2.515140001 .32675600-0.95651500$ O $1.220848000 .73085000-1.78049600$ O $3.847962001 .27271900-1.90712100$ H $3.828842001 .77664900-2.72610000$ O $2.086966002 .85345100-0.48686100$ H 2.653183003 .307896000 .14453800 O $0.90370500-1.261228001 .00242900$ Si - $0.12578400-1.81294300-0.20343600$ O - $1.21151000-0.66238700-0.46688700$ O $0.84938800-2.10586000-1.51995700$ O - $0.77616900-3.245269000 .26127800$ Si -3.02011100 -0.42149500 -0.42103600 H -1.73294400 -3.09144500 0.40404000 O $-3.01383800-0.23807000-2.10511400$
O -2.790902001.00604300 0.47047500

H -2.11163900 -0.26019500-2.43770900 Si - 1.471273001 .848783000 .90157300 O -0.67818400 $2.33869600-0.47210500$ H $0.113079002 .88098000-0.32321000$ O - 1.930362003 .245048001 .66342600 O -0.473128001.084087001.96763900 $\mathrm{H}-0.076121000 .248190001 .67560900$ H - 2.270085003 .152541002 .55762600 H $0.352946001 .03210900-1.44350700$ H $3.06584600-1.773514003 .04996900$ H 1.04786500 -1.27685200 -1.98576200 O $-4.75989300-0.32102000-0.34770800$ H -5.10118600 $0.47815500-0.75599100$ O $-3.12471800-1.922741000 .39535000$ H -4.04678700 -2.14126400 0.56307100 TS2

Si $2.50167900-1.013227001 .07458900$ O 2.901496000 .408097000 .36395800 O $2.99028700-0.847943002 .62480700$ O $3.26137000-2.288019000 .37130500$ H $2.81261600-2.62138100-0.41929900$ Si $2.548640001 .28002800-0.97179800$ O $1.296414000 .63473300-1.82043900$ O $3.907532001 .24621100-1.88360200$ H $3.904513001 .74755500-2.70438600$ O $2.072741002 .80477100-0.53956600$ H 2.616383003 .288237000 .09031200 O $0.86466400-1.210977000 .98264600$ Si -0.09091600 - $1.83584500-0.24179000$ O - $1.10574800-0.66003100-0.70802200$ O $0.95128800-2.25606600-1.46269700$ O -0.87860900 -3.16278900 0.28742700 Si -2.81839600 -0.45229000-0.47683100 H - $1.83639400-2.914964000 .38533700$ O -3.17637600 - 1.855392000 .30719000 O $-3.21428300-0.21028100-2.08178900$ O - 2.731324000 .973712000 .39890700 H $-2.42895300-0.25587000-2.63577300$ Si - 1.463807001 .899758000 .84451100 O -0.67540600 $2.37466600-0.52656900$ H $0.168180002 .84037400-0.40005100$ O - 2.008777003 .269866001 .58386200 O -0.465052001 .157271001 .92300400$ 
H -0.064307000 .314950001 .65390100$ H -2.39982200 3.165741002 .45569800 H $0.401251000 .87731700-1.51982300$ H 2.88216900 -1.60559400 3.20689800 H 1.18833200 -1.48341400 - 1.99888800 O $-4.90211100-0.43450800-0.10053300$ H -5.11591200 0.18675200 0.60323700 H $-4.31372500-1.384200000 .32218800$ Product

Si 2.23554800 -1.12490600 1.10937600 O 2.945457000 .204702000 .47235500 O $2.41589600-0.981438002 .72587700$ O $2.95129700-2.505708000 .58608900$ H $2.71501300-2.76603500-0.31452700$ Si $2.685154001 .31326300-0.70003300$ O $1.512573000 .83454500-1.74795800$ O $4.120800001 .43924800-1.47438600$ H $4.186783002 .08650000-2.18266400$ O $2.156286002 .73694000-0.04010900$ H 2.65476100 3.107167000 .69519100 O $0.63427600-1.116203000 .69188500$ Si - $0.12636000-1.72979000-0.66626400$ O - $1.08052800-0.54223700-1.25929500$ O $1.06761400-2.13608800-1.73241300$ O $-0.99938300-3.05097100-0.28108900$ Si - $2.73522700-0.37351600-0.94559900$ H - $1.94176600-2.75191800-0.14504100$ O -3.24579700 - $1.60967100-0.09847600$ O -3.52029100 -0.19477500 -2.39407000 O $-2.808125001 .11366700-0.18987000$ H -3.33087200 $0.61602900-2.87503500$ Si - 1.659313001 .911606000 .65710800 O -0.55741200 $2.47835800-0.42546500$ H $0.279739002 .83685200-0.08403700$ O -2.32495100 3.193655001 .44054400 O -0.96587900 0.935917001 .79817400 H -0.365569000 .241934001 .47165900$ H -2.99615500 3.006881002 .10311200 H $0.602444001 .10861400-1.53931800$ H 2.10703100 -1.70715200 3.27607500 H $1.43343400-1.35872100-2.17962500$ O -2.98534300 -1.12938200 2.58034400 H -2.34584500 -0.40430100 2.56711000 H -3.12816700 -1.33350200 1.62576500

$$
\mathbf{T}_{4}^{-}+\mathbf{T}_{2} \rightarrow \mathbf{T}_{6}^{\prime-}+\mathbf{H}_{2} \mathbf{O}
$$

Reactant

O -3.33350600 -1.21215100-0.22734000

Si -3.398164000 .382910000 .00126600$

O -1.06089900 -2.03659000 -0.56056600

Si $0.10382700-0.96763700-0.49410700$

O $1.63742400-1.53085500-0.73325500$

O - 2.504645000 .765684001 .35171400

O $-2.730781001 .14406600-1.31940300$ O $0.19827500-0.178574000 .99117000$

O -0.01370700 $0.22738100-1.67047500$

O -4.958091000 .854069000 .19777200$

H -2.49166800 1.685879001 .63459900

H -2.75571700 $2.10623900-1.34201300$

$\mathrm{H}-0.673188000 .107156001 .31823800$

H $-0.918092000 .57885800-1.75008700$

Si $2.92179500-2.133529000 .05262700$

O $4.03289300-2.68702900-1.04244400$

H $3.73524900-3.27834000-1.73983500$

O $3.74328900-0.974179000 .86995300$

O $2.37343400-3.281047001 .08829400$

H $3.01986900-3.722663001 .64672100$

Si 4.818225000 .176861000 .37803100

O 5.554749000 .861134001 .67369500

H 6.110189000 .293423002 .21552600

O $4.033385001 .37162900-0.40502700$

O $5.92877300-0.51689100-0.61592900$

H $5.61092900-1.32385800-1.04801100$

H -2.34575200 -1.63017400 -0.38052300

Si -6.37525000 0.01496000 0.22510500

O -6.09395000 - $1.56399700-0.12034800$

H -5.14456700 - $1.77896700-0.18987100$

O $-7.425505000 .54523400-0.92619800$

$\mathrm{H}-7.877401001 .37663100-0.75553900$

O -6.978340000 .267912001 .73752300$

H -7.85732300 -0.07303900 1.92650600

Si $2.714679002 .33796700-0.19165900$

O $3.344650003 .85035400-0.02904400$

H 2.714676004 .571797000 .05170500

O $1.770930002 .28850800-1.52787900$

H $1.130267001 .54920300-1.61263200$

O 1.912653001 .932023001 .17820100

H 1.302701001 .162942001 .14421400 
TS1

O -3.77959400 - $1.61920000-0.39233600$ Si -3.32767500-0.04966600-0.15946500 O - $1.26885400-1.54044500-0.53603300$ Si $0.07916200-0.72895100-0.48372500$ O $1.50460600-1.53880800-0.73264800$ O -2.49459600 0.268973001 .24701300 O -2.65970400 $0.73529100-1.46615700$ O 0.306550000 .024331001 .00769900 O $0.142324000 .46813300-1.66580800$ O -4.78846700 0.719426000 .04904400 H -2.96645200 0.868159001 .83508800 H -3.19599100 $1.47260100-1.77646000$ H -0.572448000 .211879001 .38210500$ H $-0.770296000 .75638200-1.84393100$ Si $2.76379300-2.164141000 .07216200$ O $3.83827200-2.81929200-1.00620100$ H $3.49997000-3.40718400-1.68784000$ O $3.65514000-1.024352000 .84312600$ O $2.17409000-3.246901001 .15574600$ H $2.80810400-3.693027001 .72479700$ Si 4.809097000 .038735000 .33571300 O 5.637235000 .636138001 .61953300 H 6.161311000 .014203002 .13234900 O $4.112704001 .31247600-0.40510300$ O $5.82504000-0.73228400-0.70232000$ H $5.44422600-1.53388900-1.09264100$ H - $2.93559600-2.10113900-0.52139100$ Si -6.33947700 0.25144200 0.28394400 O -6.55946000 - $1.29641500-0.22363700$ H -5.72023600 - $1.77382900-0.33525900$ O $-7.394479001 .14692700-0.60958600$ H -7.59878000 $2.02752300-0.28226100$ O -6.606935000 .462921001 .89983000$ H -7.48628500 0.25805000 2.23044100 Si $2.909016002 .40563700-0.13026600$ O 3.690544003 .842806000 .05674300 H 3.138904004 .619881000 .18200400 O $1.935551002 .48959000-1.44315600$ H $1.273284001 .77140000-1.55937800$ O 2.100466002 .042389001 .24802300 H 1.441998001 .312575001 .20513900 Intermediate O $2.799307001 .02198700-0.85989500$
Si $3.02009800-0.66537200-0.81127500$ O $1.41512600-0.97470800-1.52658600$ Si - $0.08659000-1.15332900-1.01815600$ O - $1.095041000 .06966500-1.47044100$ O $2.51594500-1.443926000 .63083800$ O $3.68979400-1.51766800-2.10434400$ O -0.13209100 - 1.266664000 .63737600 O -0.90010900 -2.44699400 -1.67850500 O $4.67550600-0.41403600-0.16336600$ H $3.14752600-1.339021001 .35561800$ H $4.64711100-1.52371800-2.00977300$ H $0.79377700-1.364748000 .95948900$ H -0.46195300 -3.30175300 - 1.61577700 Si -1.33050200 1.62106700-1.04417400 O $-2.297793002 .37399700-2.14556900$ H -2.06189500 $2.33197500-3.07699900$ O -2.16272500 1.743066000 .35968000 O $0.170055002 .27944600-0.89414400$ H $0.248251003 .18952100-0.59204800$ Si -3.76901600 1.497327000 .66249600 O -4.107930001 .808004002 .23353700$ H -3.98923300 2.710414002 .54377100 O $-4.14368800-0.060636000 .37818500$ O $-4.653502002 .47944500-0.31568200$ H $-4.235976002 .66329500-1.16881600$ H $1.896859001 .31339500-1.05168400$ Si 5.168509000 .500458001 .07045600 O 4.613575002 .049335000 .93994600 H 3.865882002 .027068000 .31005400 O 6.810714000 .649272001 .19084700 H 7.28286500 -0.12168600 1.51741200 O $4.56503400-0.267984002 .42673700$ H 4.582453000 .207611003 .26170400 Si -3.77916400 - 1.617791000 .76171000 O $-5.14575800-2.187051001 .47958000$ H -5.15029800 -3.11675100 1.72347900 O $-3.51150100-2.47826600-0.60882300$ H -2.62278600 -2.49307400 -1.00908600 O $-2.53522800-1.689703001 .82480200$ H - $1.62676800-1.548218001 .48448000$ TS2

O -2.60622600 $1.26199200-0.10705800$ Si -2.90505100-0.03900400 0.89971100 O - $1.49047200-0.598789001 .60021800$ 
Si $0.06539700-0.829277001 .11851400$ O 0.899610000 .590313001 .37372300 O - $2.38844400-1.44870800-0.52968500$ O -3.45268800 0.550640002 .39502900 O $0.15196100-1.34463000-0.38642400$ O $0.82085300-1.815635002 .24317200$ O -4.46602600 -0.51245300 0.40494800 H -2.90618800 -1.42813100 - 1.35151900 H -4.39061600 0.758545002 .36589900 H - 1.39175100 -1.47146700 -0.69631900 H $0.37698900-2.653051002 .41174300$ Si 1.315027001 .869274000 .47134400 O 2.205539002 .932095001 .36835200 H 1.892222003 .167303002 .24668200 O $2.296989001 .49982000-0.78306100$ O -0.09360600 $2.51508800-0.10523200$ H $-0.032593003 .25762500-0.71473300$ Si $3.911807001 .16249600-0.85704400$ O $4.406271001 .08877500-2.41743400$ H $4.354689001 .89757400-2.93480100$ O $4.20541000-0.28963900-0.18484500$ O $4.733184002 .33855400-0.05203700$ H 4.212649002 .779569000 .63469000 H - $1.737070001 .69614400-0.07050400$ Si -5.28385600-0.12078600-0.94932500 O -5.14373300 $1.47934300-1.30481800$ H $-4.259918001 .80070900-1.05563200$ O $-6.90076400-0.37457800-0.80515000$ H -7.20342700 -1.28664600 -0.82820000 O $-4.61583000-1.08695300-2.13121200$ H -4.83648600 -0.89417700 -3.04710200 Si $3.70751100-1.86264400-0.27252200$ O $5.03305900-2.66296600-0.83905500$ H 4.95123900 -3.61643300 -0.92762000 O $3.39540900-2.401731001 .24954400$ H $2.52168100-2.210592001 .63970100$ O $2.45468800-2.03908600-1.29550300$ H $1.53540500-1.76942000-0.96953800$ Product O $2.45502900-1.79977100-0.58781900$ Si $2.95653900-1.130043000 .83546700$ O $1.72708800-0.490070001 .68656000$ Si 0.260371000 .162666001 .25709400 O - $0.79862400-1.118768001 .15532800$
O 1.831978003 .307542000 .11614200

O $3.65993600-2.199862001 .86407300$ O $0.307550001 .05463200-0.04691300$ O -0.327979000 .931594002 .62430500$ O 4.057743000 .003444000 .38466200 H $2.707654003 .02437800-0.18489700$ H $4.47965700-2.609668001 .57292500$ H 1.274543002 .498635000 .05392800 H 0.240198001 .623537002 .97863000 Si -1.41520900-2.06968400 0.00023600 O -2.46077700 -3.16555900 0.65564600 H -2.18549100 -3.65670700 1.43537700 O -2.32513100 -1.26465400 -1.09320900 O - $0.12588400-2.77521900-0.76197500$ H -0.30677600 -3.36328700 - 1.50258200 Si -3.86030600 -0.65913900-1.00737200 O $-4.34522600-0.13049000-2.48046700$ H $-4.43786400-0.78758100-3.17629000$ O -3.899021000 .612940000 .00527000$ O $-4.86553600-1.84447400-0.46816600$ H -4.42674400 -2.51736500 0.07161800 H $1.57269400-2.21581800-0.63960800$ Si $4.582661000 .66124500-1.02221300$ O $3.723251000 .06492700-2.28431400$ H $3.11207700-0.64555900-2.02529800$ O $6.162199000 .31372100-1.32458800$ H $6.820953000 .73640200-0.76584400$ O $4.380355002 .29155900-0.83064400$ H $4.817573002 .86765000-1.46531800$ Si -3.138321002 .057526000 .26235800$ O -4.30194200 $3.17931400-0.06341600$ H -4.04710100 4.10040000 0.03875400 O -2.74931700 2.17007800 1.85608200 H - 1.927546001 .745853002 .16659600 O - $1.867020002 .24718000-0.73473800$ H - $1.003487001 .77863100-0.49053300$

$\mathbf{T}_{3}^{-}+\mathbf{T}_{3} \rightarrow \mathbf{T}_{6}^{\prime \prime-}+\mathbf{H}_{2} \mathbf{O}$

Reactant

O - $1.50363400-1.190849001 .61784600$ Si -2.09953500 - 1.583009000 .17002100 O $0.99949100-1.128644001 .49674800$ Si $1.67859500-1.727648000 .19887600$ O $3.34544800-1.659252000 .16794200$ 
O - $1.54679500-0.62068300-1.06337900$

O - $1.60574900-3.11760800-0.21854800$

O $1.27876000-0.89550900-1.21672500$

O $1.36291100-3.34688800-0.06000800$

H - $1.847762000 .30930700-1.05553700$

H - $1.86298600-3.44429800-1.08674400$

H $0.33407200-0.67226900-1.28987000$

H $0.41180300-3.53029100-0.03223100$

Si $4.44986200-0.78096700-0.63504200$

O $3.709397000 .12193800-1.81282700$

H $2.75171800-0.08301900-1.86613600$

O 5.235409000 .284117000 .34325900

O $5.55368900-1.84140900-1.23051100$

H $6.39992200-1.48163000-1.51078700$

Si 5.060623001 .888183000 .65492800

O 6.499590002 .601992001 .00698100

H 7.069616002 .820120000 .26396600

O 4.141153001 .978747002 .01494000

H 4.007357002 .848347002 .40219600

O $4.390278002 .62605100-0.65620900$

H $3.993393001 .99412000-1.28017100$

O -3.74488800 -1.53645300 0.21772500

Si -4.97056100 -0.47976000 0.37210000

O -4.96802200 0.276444001 .84299000

O $-4.817811000 .76629900-0.68550900$

O -6.31962700 - 1.353546000 .07119300

Si -4.12747700 $2.25350400-0.61033100$

H -7.16691200 -0.90537800 0.14802400

O $-4.673160003 .25072000-1.78592900$

O -2.52663400 $1.96565200-0.92706700$

O -4.386690002 .932392000 .86151400$

H -5.52755200 3.67115500 -1.65099300

H - $1.906156002 .69801200-0.85859800$

H -4.53806400 2.28488500 1.56704800

H -4.92078800 -0.25748800 2.64150000

H $-0.46368400-1.143906001 .62518000$ TS1

O $1.35770600-0.70865300-1.77914700$

Si $1.56877400-1.50952500-0.34596800$

O $-0.90614100-1.28721300-0.88656400$

Si - $1.83313800-1.918442000 .22522100$

O -3.48040300 - 1.680995000 .06430900

O $1.13331400-0.712015001 .03759500$

O $1.12593800-3.10567700-0.26601800$
O - $1.52591900-1.236666001 .74324000$

O -1.69998800 -3.57594400 0.34128900

O $3.24415800-1.58585900-0.29032200$

H $0.38172100-0.64617700-1.86650000$

H 1.720031000 .018471001 .30960600

H $1.86877900-3.69014100-0.08171300$

H $-0.61463400-0.892353001 .74423100$

H - $0.78857500-3.816850000 .11765600$

Si -4.45908100 -0.48207200 0.56281300

O -3.786185000 .273932001 .87707600$

H -2.91866300 -0.12787500 2.10036000

O $-4.678058000 .67531900-0.58715600$

O $-5.91562500-1.163264000 .89085800$

H -6.68026500 -0.58078800 0.89885600

Si $4.42347200-0.55633500-0.73184800$

O $3.878372000 .43096300-1.94669700$

O $5.72357900-1.45780300-1.14923800$

H $6.58143800-1.02426200-1.16302300$

Si -4.00474300 $2.15443800-0.85952500$

O -3.519910002 .797629000 .57132100$

H -3.48916600 2.14015500 1.28694800

O $-2.683897002 .06151100-1.83203600$

H -2.82048100 $1.76882900-2.73760900$

O $-5.092160003 .11642800-1.63026100$

H -5.96670900 $3.19250300-1.23768300$

O 4.844416000 .440307000 .51363000

Si 4.201012001 .874633000 .98355800

O 5.111364002 .629484002 .11360000

O 2.767620001 .467478001 .71261500

O $4.005758002 .85770700-0.31822500$

H 5.879860003 .120858001 .80907100

H 2.228354002 .186654002 .05763400

H $3.873563002 .36434300-1.14331500$

H $2.965097000 .21552300-2.21299500$

Intermediate

O -2.97999600 2.470155001 .98098500

O -3.59671600 - $0.79885900-2.37302400$

Si -2.127683001.203202001.25021100

Si -3.32961200 -0.82453700-0.74590200

O $-2.703201000 .57349100-0.23488200$

Si $2.827075001 .46779000-0.09774100$

Si $3.25039000-1.588108000 .47364200$

O 2.934876000 .004332000 .61752100 
O -0.65348300 0.568561001 .87500500

O -4.72794600 - 1.091837000 .09732200

O 4.059312002 .340049000 .54383200

O $2.46829600-2.421615001 .63973000$

Si $-0.69221300-2.30551500-0.31339800$

Si $0.023337002 .46507400-0.65102300$

O $0.06364900-1.35699000-1.43710900$

O $0.232735003 .92231900-1.39563800$

O $0.049567001 .41720300-1.93288400$

O - $2.29638500-2.08257900-0.51903300$

O - 1.237846002 .443401000 .32892300

O 1.363130002 .102205000 .28775400

O -0.21099000 - 1.952631001 .22098100

O $4.85013500-1.940527000 .55398600$

O $2.992319001 .39004100-1.73373400$

O - $0.27240200-3.86462000-0.61326000$

H $5.23734000-2.058703001 .42596800$

H $2.152797001 .29743700-2.20942300$

$\mathrm{H}-4.18969000-0.11652400-2.70052000$

H $-0.40041700-4.501016000 .09589400$

H $0.096704004 .69924300-0.84651800$

H 4.154986003 .247070000 .23912700

H 0.125582001 .081122001 .62113900

H -0.12981500 $0.47861100-1.73605300$

H $1.03041400-1.50480900-1.46886200$

O $2.73895800-1.96983500-1.06178300$

H $2.93902600-2.85724300-1.37748500$

H $1.49449200-2.311021001 .63331200$

H -0.41211300 -1.02903200 1.53972100

O -2.92686100 -0.03012100 2.21893800

H $-3.80640500-0.267225001 .90651200$

H -3.56510600 2.10518800 2.65135100

H -5.22313300 -1.89094100 -0.10423100

TS2

O -2.84743000 2.29813000 2.11793800

O -3.70396000 -0.47663200 -2.33447600

Si -1.909743001.453374001.09752900

Si -3.40124300-0.66493800 -0.72722800

O $-2.651285000 .63759400-0.12986100$

Si 2.915356001 .339219000 .02447600

Si $3.11378800-1.740866000 .47344500$

O $2.81036600-0.149510000 .68492600$

O -0.64252900 0.551930001 .73939000

O $-4.78400700-0.906800000 .13307500$
O 4.144739002 .084527000 .80491100

O $2.28944100-2.611541001 .58093100$

Si $-0.83315500-2.29103700-0.43191400$

Si $0.243740002 .53114000-0.71705600$

O -0.00722400 -1.31966900-1.48444800

O $0.563463003 .95299400-1.46600200$

O $0.186215001 .45197600-1.95651100$

O -2.42191600 -1.97631600-0.63730600

O -1.08281200 2.624193000 .21284700

O 1.471012002 .078480000 .31154000

O -0.37455400 -2.03659600 1.13159200

O $4.70535500-2.119506000 .56965900$

O $3.226469001 .31084900-1.59001400$

O - $0.48223400-3.84798900-0.80591800$

H $5.08201000-2.256562001 .44358900$

H $2.458897001 .17267000-2.16104400$

H $-4.303124000 .23348900-2.58257600$

H $-0.68449700-4.52105500-0.14974700$

H $0.586132004 .74366200-0.91985000$

H 4.358632002 .983944000 .54068500

H 0.224970000 .954307001 .58329800

H - $0.042077000 .52362000-1.75743900$

H $0.95107800-1.51951200-1.50920000$

O $2.63288300-2.03841200-1.08987800$

H $2.83817800-2.90669000-1.45240400$

H $1.31960200-2.478632001 .56574600$

H - $0.53011700-1.117158001 .46165400$

O $-3.21301500-0.012460002 .62410400$

H -3.96841600 -0.40007600 2.16865500

H -3.24927300 1.420324002 .56417900

H -5.39623000 - $1.57078800-0.19706400$

Product

O -2.886164002.227064001.96867200

O -3.97038200 -0.55999800 -2.16677700

Si - 1.896934001 .520553000 .98284800

Si -3.33362400 -0.85971500-0.68164200

O $-2.613806000 .41633800-0.02498600$

Si 2.958052001 .392738000 .11325900

Si $3.16700600-1.663941000 .69560100$

O $2.81462800-0.073707000 .81744900$

O -0.66438600 0.593886001 .69538700

O $-4.48756600-1.346683000 .39816400$

O 4.116644002 .178338000 .95986300

O $2.25882500-2.512743001 .75448900$ 
Si $-0.66422600-2.31091200-0.56752300$ Si $0.322000002 .46963300-0.86815700$ O $0.24627600-1.38450200-1.59035300$ O $0.628942003 .87741200-1.65212100$ O $0.395559001 .34963700-2.06727400$ O -2.23171100-2.04559400-0.94642700 O - $1.082207002 .54753400-0.05626300$ O 1.492532002 .128656000 .25971900 O -0.34805400 - 1.964071001 .01315200 O $4.74968000-2.003696000 .95183600$ O $3.394872001 .30793300-1.46998000$ O -0.25541900 -3.87652900-0.83187200 H $5.05276600-2.070979001 .86197400$ H 2.67407900 1.14714200 -2.09372100 H -4.69728500 $0.06798500-2.21134400$ H - $0.54992800-4.53100400-0.19204000$ H $0.580760004 .68623100-1.13499700$ H 4.339147003 .072073000 .68360600 H 0.204993001 .015361001 .70316600 H $0.192528000 .41487300-1.86480000$ H 1.20487800 -1.56775500 - 1.50490700 O $2.83919200-2.03115800-0.89247400$ H $3.10020100-2.90539500-1.20041200$ H $1.29356600-2.400602001 .63861900$ H -0.49564100 -1.02575400 1.29713900 O -5.16660100 0.805353002 .31961400 H -5.04337000 0.051545001 .72581900 H -4.34488500 1.346114002 .20690000 H -5.09047600 -2.04456700 0.12505400

$\mathbf{T}_{3}^{-} \rightarrow \mathbf{R}^{-}+\mathbf{H}_{2} \mathbf{O}$

Reactant

Si - $1.98709900-0.64634700-0.00626400$ O - $1.317567000 .83635200-0.43408200$ Si $0.090651001 .58813800-0.11629400$ O $1.373324000 .70442300-0.66698100$ O -0.87971900 - 1.685482000 .46285300 Si $1.93924500-0.73208800-0.07518300$ O 0.306423001 .870269001 .49940400 O $0.004429003 .02410800-0.90839500$ O $1.42430900-0.867488001 .49580400$ O $3.57378100-0.61850300-0.17729800$ O -2.91443400 -1.07406400-1.32492900
O -3.17007300 -0.33762600 1.12891500 H 0.729480001 .122629001 .94764900 H $0.749609003 .62229800-0.80347800$ H $0.50319900-1.236469001 .42982300$ H - $2.44531600-1.08820400-2.16344600$ H $-2.91358500-0.574330002 .02406500$ O $1.38087600-2.00501900-0.95300700$ H $0.44817600-2.14227800-0.65287500$ H $4.07849600-1.415245000 .00836400$ TS1

Si - $1.79431200-0.742466000 .01481900$ O - $1.608093000 .90235400-0.26896600$ Si -0.14841600 $1.63489500-0.13144700$ O $1.035235000 .67505700-0.74023300$ O -0.36845700 - 1.359808000 .37850800 Si $1.70870500-0.69065700-0.05663200$ O 0.222925002 .008530001 .43904100 O $-0.296056003 .03147800-0.98632000$ O $1.71621900-0.663265001 .62383500$ O $3.32151400-0.27668100-0.34772300$ O - $2.57650000-1.38573500-1.30461200$ O -2.96785600 -0.94183000 1.17534200 H 0.794762001 .352967001 .86358000 H $0.421519003 .66524400-0.90060800$ H $0.87487000-1.046597001 .91025200$ H -2.08575200 - $1.34089200-2.12966900$ H -2.65427900 -0.84905300 2.07896500 O $1.67032900-2.12760400-0.89692400$ H $0.79121600-2.50886500-0.76789900$ H $3.93989000-0.99757900-0.20457600$ Intermediate Si $1.07242100-1.45348700-0.07691000$ Si 0.999833001 .453517000 .02662100 Si -1.66114000 -0.01917300 0.12280300 O -0.388674001 .095343000 .77428400$ O $-0.54064900-1.29516800-0.08388800$ O $1.801972000 .02180200-0.23914400$ O 2.051590002 .356351000 .92275100 H 1.867838003 .298586000 .96834100 O $0.709451002 .24331000-1.39923400$ O $1.65172400-2.207936001 .27763900$ O $1.46132900-2.44063900-1.33869000$ O - $1.790435001 .04764300-1.21689200$ H $2.36709100-2.76052500-1.37159800$ 
H -2.46918900 $0.73163800-1.82149300$ H -0.20222100 2.02134400 - 1.66872600 H $1.66468200-1.685177002 .08453400$ O -2.423052000.064554001.63768000 O -3.00196500 -0.92951100 -0.52936900 H -3.35866900 - 1.554060000 .10667500 H - 1.875461000 .570220002 .24598100 TS2

Si $1.74903100-0.850730000 .07803900$ Si $0.256753001 .73410400-0.06261700$ Si - $1.30796500-0.86659700-0.08262300$ O - $1.029637000 .76079800-0.31731400$ O $0.26423000-1.49686400-0.05002700$ O 1.597393000 .795322000 .10292100 O 0.157271002 .602735001 .32796600 H - 0.498713003 .304826001 .35981500 O $0.399623002 .81373100-1.29537600$ O $2.57015300-1.339615001 .41870600$ O $2.62142200-1.36098600-1.21680100$ O - $1.92630200-1.58941100-1.44185100$ O - $1.91934000-1.288160001 .37161900$ H $3.57352500-1.23300300-1.18226400$ H - $1.25694900-2.09855300-1.90981200$ H $0.242539002 .48250900-2.18450900$ H $2.27713200-0.984206002 .26288600$ O -3.68628700 -0.11234300 0.22957300 H -2.84132600 -0.82988600 1.21193900 H -3.65388200 0.841780000 .34743300 Product

Si - $1.61482500-1.21744600-0.03002100$ Si - $0.768573001 .63823200-0.10430900$ Si $1.28844000-0.41419200-0.06239500$ O $0.629932001 .00325800-0.68595000$ O -0.00948400 - $1.49170800-0.11223000$ O - $1.877764000 .41507100-0.07031200$ O - $1.407538002 .79697400-1.06864200$ H - $1.008471003 .67072500-1.03064200$ O -0.50952200 2.26298300 1.40006600 O -2.45264500 - $1.93696100-1.25160500$ O -2.15852700 - 1.883363001 .37038700 O 1.418939000 .048154001 .55557000 O $2.57719100-0.95152300-0.76878300$ H -3.11098800 -1.93991700 1.48811800 H $1.75115600-0.614384002 .16714400$
H 0.147810001 .739773001 .88510600 H - $2.37659400-1.54033500-2.12432800$ O $5.10473100-0.42441300-0.21133200$ H $4.14499300-0.60224300-0.41295600$ H $5.259004000 .48627600-0.47564500$

$\mathbf{T}_{4}^{-} \rightarrow \mathbf{R}_{\mathbf{4}}^{-}+\mathbf{H}_{2} \mathbf{O}$

Reactant

Si 1.542950001 .698672000 .13984100 O $0.362530002 .61849200-0.37433400$ O $1.799196000 .34948900-0.82379900$ O 3.055624002 .387892000 .21460400 Si -2.16848500 $1.04301000-0.18572000$ Si $1.81841400-1.24331100-0.46299900$ H 3.063675003 .313944000 .47106000 O -1.37277900 -0.13066200 0.67839700 O $0.29684700-1.87033000-0.52040000$ O -3.69166500 $0.42114200-0.33870200$ O - 2.115887002 .433464000 .68147900 O $2.63126700-2.11404900-1.59087300$ O $2.49694100-1.475486001 .02347300$ Si -1.09113300 - 1.715015000 .32352700 H -4.29437100 $0.86225600-0.94459100$ H - 1.218567002 .809320000 .50929400 H $3.54955400-1.87549200-1.74526700$ H $2.27724600-0.748208001 .62850600$ O -2.33844000 - 2.30243900 -0.57254100 O $-0.86808200-2.564361001 .71158900$ H -3.09125000 -1.70323200 -0.66980000 H -1.62061500 -2.63977500 2.30534600 O 1.290254001 .056512001 .68322300 H 0.361388000 .845256001 .84285400 O - $1.515548001 .23995600-1.68017400$ H -0.69356400 $1.77198000-1.53800800$ TS1

Si 1.056049001 .860503000 .20942500 O -0.42182500 $2.30294100-0.15276400$ O $1.674972000 .68336100-0.80860400$ O 2.220151003 .045470000 .12015100 Si -2.19995700 $0.69636900-0.24332600$ Si $2.07165100-0.87498000-0.51201600$ H 1.944754003 .906390000 .44675700 O - $1.16450200-0.275507000 .62895200$ O $0.72370700-1.81890500-0.49001100$ 
O -3.48028800 - $0.40095500-0.40085900$

O -2.85576800 1.918359000 .65143700

O $2.97509500-1.51594000-1.72157900$

O $2.89149200-0.977761000 .91642500$

Si - $0.66257200-1.822501000 .37971100$

H -4.14642300 - $0.16564400-1.05288500$

H -2.16025500 2.589553000 .73742700

H $3.82068700-1.09631200-1.90302100$

H $2.56032200-0.326478001 .55643300$

O - $1.82754600-2.65919900-0.41829200$

O $-0.27097200-2.520897001 .81698200$

H -2.62060300 -2.11133400 -0.56655100

H -0.99326300 -2.68033200 2.43134600

O 1.197215001 .198978001 .75686100

H 0.360803000 .806666002 .03706100

O - $1.831757000 .94979100-1.83806100$

H - $1.118202001 .60812000-1.84479900$

Intermediate

O - $1.45098500-1.17913100-0.76497600$

O $1.205966001 .23265200-0.57595800$

Si 0.004457002 .121293000 .06335900

Si $2.13161000-0.15423300-0.23360800$

Si -2.32034800 $0.11916200-0.24546700$

Si - $0.26145600-2.04679200-0.00282000$

O $-0.50653800-3.64706200-0.28540000$

H - $0.72522500-3.90710400-1.18450200$

O $-0.42102600-1.804464001 .62641300$

O -2.77153200 -0.13861600 1.31885100

O -3.64222400 $0.36107700-1.18460400$

O 0.138915002 .171167001 .69914200

O $0.040303003 .68143900-0.46364900$

H $0.31157000-1.192131001 .88567800$

H -0.34045300 $3.85010900-1.32988000$

H -4.31114700 -0.32922200 - 1.18389000

H -2.13891700 -0.70163100 1.79467300

O $1.19699000-1.55259200-0.50900300$

O -1.44839600 $1.49014100-0.41009900$

H 0.704229001 .405703001 .95105800

O $1.59187300-0.070358001 .52842200$

H $2.35620900-0.233761002 .08961100$

O $3.69619800-0.281273000 .42153400$

H $4.34599400-0.32647300-0.28672900$

O $2.85022900-0.15321000-1.82145600$

H 2.19984400 $0.00433800-2.51023500$
TS2

O - $1.95598100-0.78661800-0.64407900$

O $1.383670000 .74648000-0.83928000$

Si $0.536622001 .95412300-0.13183200$

Si $1.84842100-0.76490300-0.32997800$

Si -2.29259400 0.72249100-0.07699600

Si -0.92191400 - $1.94439800-0.08442300$

O - $1.54904600-3.42271500-0.40723400$

H -1.94822100 -3.55751700 -1.27135900

O $-0.82506800-1.776223001 .56001000$

O $3.05756300-1.42890100-1.22057600$

H $3.84182300-0.95136900-0.87157100$

O -2.55337300 0.625894001 .54579400

O $1.80870900-0.860668001 .33894900$

O -3.59267600 $1.36745300-0.83300300$

O 0.830760001 .984354001 .48592700

O $0.977765003 .42271700-0.70753400$

H $0.07709300-1.559712001 .85712700$

H $0.522308003 .74225300-1.49121900$

H $-4.441026000 .93544900-0.69887700$

H -2.08660900 -0.11376600 1.96391900

O $0.55217500-1.74581300-0.74502600$

O -1.06458700 $1.73898000-0.44113100$

H 1.093085001 .124981001 .84613000

O 3.912183000 .156607000 .57801300

H 3.988780001 .112252000 .50742400

H $2.70853400-0.447329001 .51699300$

Product

O - $1.78751600-1.17337400-0.58006700$

O $0.956027001 .19087200-1.07716900$

Si -0.05716400 $2.11581700-0.16744100$

Si $1.77493300-0.16524600-0.54227500$

Si -2.42498700 0.157492000.15665500

Si $-0.43636100-2.05237700-0.21687800$

O -0.71237900 -3.62721000-0.56977400

H -1.36957400 -3.82099200-1.24391600

O $-0.12621600-1.893235001 .39721600$

O -2.40863600 -0.08940200 1.78460600

O -3.94729200 $0.45148100-0.36803000$

O 0.494596002 .143580001 .37531500

O -0.11909400 $3.66165900-0.71414200$

H $0.66840500-1.305873001 .52233900$

H $-0.783873003 .86217400-1.37868700$

H $-4.61436500-0.21325800-0.17458800$ 
H - $1.70210800-0.693282002 .06458200$ O $0.84317600-1.45941700-1.05363100$ O - $1.579767001 .49027300-0.26864400$ H 1.068968001 .350841001 .52404700 O $4.56837500-0.291147001 .16065300$ H 4.971561000 .518632001 .48646900 H $3.59988100-0.214417001 .34716700$ O $1.88577100-0.157148001 .05953300$ O $3.20983600-0.24766800-1.35119300$ H $3.95617000-0.25754200-0.72563600$

$\mathbf{T}_{\mathbf{5}}^{-} \rightarrow \mathbf{R}_{\mathbf{5}}^{-}+\mathbf{H}_{\mathbf{2}} \mathbf{O}$

Reactant

Si -2.47551700 -0.88023900 0.97742700 O -1.67232200 2.518617000 .93328300 O - $1.09255000-1.420423000 .27561900$ Si -0.33069600 -1.93290100-1.07515800 O $1.24035600-2.20434200-0.66098600$ Si $2.43172500-1.437317000 .15074600$ O $2.498804000 .13823100-0.27144400$ Si 1.929198001 .574073000 .31367600 O $0.593531001 .97719900-0.49524000$ Si -0.99182100 $2.49218100-0.49419800$ O $2.17319600-1.408474001 .78161300$ O $3.84930400-2.20428700-0.14410600$ H $3.97794600-2.57341600-1.02263000$ O 3.017887002 .768015000 .00212500 O 1.709124001 .419758001 .93959300 O -0.99898400 $3.92956300-1.33869400$ H -0.61951900 4.67968100 -0.87304400 O - $1.724047001 .43316400-1.57652000$ H - $2.522187001 .01238500-1.21094500$ O -2.20785600 0.327781002 .00850800 O -3.10667700 -2.21058200 1.72059600 O $-0.94192700-3.34783500-1.64897400$ H -0.74904700 -4.14086700 - 1.14073300 O -0.46108400 -0.85806900 -2.29142900 H - $0.837303000 .02346000-2.06980700$ H -3.88729100 -2.06888400 2.26377000 H 3.866655002 .702777000 .44917800 H - 1.974015001 .255254001 .58812800 O -3.49659200 - $0.31050200-0.21847200$ H 1.728357000 .504211002 .25682700 H $1.96611800-2.230811002 .23448000$
H -3.87143400 -0.96748600-0.81391100 TS1

Si -2.39376100 -0.75344300 0.87193400 O - 1.812230001 .685211000 .88209100 O -0.85840100 - 0.904378000 .28093700 Si -0.24831900 - $1.78121100-0.96619300$ O $1.31219600-2.17032200-0.60543000$ Si $2.52308600-1.342471000 .11761200$ O $2.491987000 .22732500-0.33035600$ Si 1.889784001 .650393000 .25469400 O $0.528585002 .03997500-0.51959000$ Si -1.12670600 $2.21410600-0.43792400$ O $2.36280900-1.299162001 .76102100$ O $3.95091400-2.05570000-0.25082800$ H 4.05067700 -2.41314400 -1.13795800 O $2.941315002 .86750800-0.09815700$ O 1.714652001 .502865001 .88707600 O -1.41587100 $3.81733800-0.81343100$ H - $1.284838004 .43207700-0.08652000$ O -1.66197600 $1.39788900-1.81170300$ H -2.48086100 $0.92721700-1.57532400$ O -2.55534600 -0.18602500 2.40147300 O -2.79492400 - 2.38827600 0.99356100 O - $1.08647300-3.18538100-1.14653600$ H - $1.77148700-3.29823000-0.46698800$ O $-0.26363300-0.90777500-2.34896500$ H - $0.68981000-0.02697000-2.26127400$ H -3.58888900 -2.62177000 1.48160000 H 3.812823002 .808916000 .30365000 H -2.31307900 0.759040002 .31915000 O -3.45034900 - $0.18763300-0.29701700$ H 1.801963000 .594910002 .21440100 H $2.22096200-2.123444002 .23507800$ H -4.00068400 -0.88951200 -0.66070100 Intermediate

Si -2.17460000 -0.69513500 0.85822100 O -2.06122800 1.082053000 .73323400 O -0.57881700 - 0.870222000 .27983400 Si $0.05322100-1.84193800-0.86551000$ O $1.67425300-2.01864400-0.56936900$ Si $2.72131100-0.944674000 .07814500$ O $2.340618000 .57958500-0.37896700$ Si 1.544565001 .878603000 .24841500 O $0.137064002 .12059400-0.52157600$ 
Si - $1.489261001 .88151500-0.54647000$ O $2.64838200-0.896890001 .72845500$ O $4.24717800-1.34534400-0.36496900$ H $4.36662400-1.71679500-1.24386700$ O $2.395839003 .25102500-0.06753900$ O 1.362329001 .663340001 .86953000 O -2.19581500 $3.36821100-0.71874600$ H - $1.978286004 .02634000-0.05279700$ O -1.83448200 $1.05077300-1.94801800$ H -2.56319700 $0.42807600-1.75470000$ O $-2.40776800-0.555079002 .52458900$ O -2.44317500 -2.45651300 0.99377400 O -0.63932800 -3.33060800 -0.81744200 H - $1.36789800-3.29049100-0.15399100$ O -0.09612800 - $1.15614100-2.35731100$ H -0.60926200 -0.32690100 - 2.35595800 H -2.35001800 -2.76265500 1.89979500 H 3.272402003 .309823000 .32293000 H -2.33800800 0.365244002 .79599300 O $-3.33535900-0.66705800-0.40150200$ H 1.636581000 .787935002 .18335000 H $2.73544900-1.719491002 .21836500$ H -3.68618500 -1.55095500 -0.55016100 TS2

Si - $1.24819000-1.301121000 .99898200$ O -2.28583200 -0.33732100 0.12785700 O $0.00299900-1.55136200-0.05369900$ Si $1.22354200-1.79001400-1.08559300$ O $2.47561700-0.82543000-0.63200300$ Si 2.604019000 .399994000 .45584700 O $1.658222001 .64433900-0.02983400$ Si 0.183205002 .344168000 .06536500 O -0.80587700 1.60526000-1.01105600 Si -2.10368500 $0.63079000-1.17248200$ O $2.23247200-0.090696001 .97248800$ O 4.140181000 .973127000 .42774900 H 4.851030000 .327884000 .47353600 O $0.268960003 .90744700-0.41495600$ O - 0.414335002 .254938001 .58732600 O -3.49828200 $1.45899100-1.41920400$ H -3.99740700 $1.71672100-0.63861200$ O - $1.77394500-0.19852700-2.56762400$ H - $2.47633600-0.75676000-2.91672800$ O - $0.49091000-0.454098002 .22378000$
O - $1.80188300-2.828018001 .26935200$ O $1.84678100-3.31152300-1.02635000$ H 1.33572800 -4.00025300 - 1.46075900 O $0.73380900-1.46011200-2.61587200$ H - $0.12386400-1.00223400-2.68859200$ H $1.29760400-0.311742002 .16182400$ H - 0.466844001 .345409001 .94523600 H -2.46708000 -2.70908700 1.97264600 H 0.921590004 .466816000 .01552600 O -2.71039700 -1.05489600 2.89299700 H - $1.30745800-0.495000002 .86178100$ H -3.41209700 -0.41213900 2.75688900 Product

Si 1.296406001 .370071001 .35238000 O 2.259703000 .446522000 .33005000 O - 0.002741001 .782444000 .41238000 Si -1.16496000 $2.01547600-0.68205500$ O -2.22963100 $0.76352400-0.64214700$ Si -2.56573000 -0.55270700 0.26738900 O - $1.26017500-1.518812000 .39831400$ Si $-0.02392000-2.23217700-0.40762300$ O $0.75602500-1.09087100-1.29684200$ Si $2.12698600-0.21068300-1.14678700$ O -2.95676500 -0.19014500 1.81914800 O -3.84646100 - $1.32610200-0.41864800$ H -3.87582700 -1.35066700 - 1.37948500 O - $0.57301900-3.29362700-1.53850200$ O $0.95144500-2.974016000 .65763100$ O $3.48325500-1.07731800-1.48149100$ H $3.90456200-1.51967100-0.73874000$ O $1.948954000 .91412300-2.35322400$ H $2.682424001 .52235800-2.48962400$ O $1.19760500-1.881201003 .03109500$ O 0.955599000 .628671002 .69977500 O -2.09003300 $3.33664900-0.34564300$ H - $1.659564004 .19221200-0.42802900$ O - $0.537725002 .18639500-2.18793100$ H $0.324978001 .75807200-2.33982100$ H $1.11169000-0.870151002 .90572400$ H -1.05099300 -4.06218200 -1.21439700 H $2.06023000-2.033699003 .42770800$ O 2.106757002 .819369001 .53711000 H $1.05098000-2.571020001 .56646400$ H -3.73727500 0.353727001 .95791500 
$\mathrm{T}_{6}^{-} \rightarrow \mathrm{R}_{6}^{-}+\mathrm{H}_{2} \mathrm{O}$

Reactant

O -2.08921600 3.494312001 .45124400

O $-4.331547000 .48469100-1.66451300$

Si - 1.451512002 .019609001 .09744900

Si -3.00620400 $0.03256700-0.81350700$

O -2.27441600 $1.32599600-0.13395600$

Si $3.43033800-0.233463000 .02083700$

Si -0.10228900 -2.11510800 1.45000800

O $3.36605700-1.088658001 .38855900$

O - 1.601470001 .176336002 .48638900

O -3.56904100 - 1.009926000 .31492600

O $4.979010000 .29050200-0.20539500$

O - $1.54743800-1.585677002 .17599100$

Si $-0.50467000-1.42038500-1.51090400$

Si $1.350425002 .19354100-0.39601300$

O $0.60823500-0.66802300-2.46076500$

O $2.166465003 .61546100-0.24756200$

O $0.832112002 .03916300-1.94224700$

O -1.93105000 - $0.67824800-1.84209700$

O 0.109519002 .196021000 .66357100

O 2.383850001 .011071000 .00551900

O - $0.15926700-1.185450000 .07347300$

O -0.43521600 -3.67206000 0.91009800

O $3.04250200-1.09387100-1.35746900$

O - $0.56408900-3.02635000-1.84543000$

H $3.56403000-1.88239400-1.53585100$

H - 1.865092004 .213141000 .85395900

H -4.18263100 $0.98879500-2.46927900$

H -0.58996800 -3.56755200 - 1.03676100

H $1.641686004 .39925400-0.06416400$

H 5.484499000 .467791000 .59284500

H - 1.554379000 .203139002 .43641600

H -2.94562100 -1.28006800 1.01496000

H $0.658237001 .12595700-2.24162500$

H $1.53330200-0.88743100-2.21176700$

O $1.18583400-1.957951002 .33115800$

H $2.47071300-1.426630001 .75487300$

H - $1.78705000-2.027120002 .99839300$

H $0.18037500-4.340257001 .22586200$

TS1

O -2.54289300 3.26307500 1.26853000
Si - 1.756295001 .878392000 .87093400

Si -3.26739100 -0.25099500-0.67393000

O -2.48372400 $1.17468000-0.42573300$

Si $3.35663700-0.02393700-0.27865700$

Si $0.70628900-1.662491001 .55170200$

O $4.30234800-0.581710000 .94439600$

O -1.860812000 .900065002 .18303000$

O $-3.76039600-0.862453000 .76739000$

O $4.366209001 .02737900-1.10538600$

O $0.57929200-0.264258002 .49230200$

Si -0.78222300 - $1.85413700-1.10358800$

Si $1.116314002 .03978400-0.45743600$

O $0.30304400-1.08474100-2.06946500$

O $1.937915003 .46128600-0.45704700$

O $0.704057001 .65134000-1.99284200$

O -2.26502300 -1.26229600-1.49712900

O -0.19594100 2.176078000 .50966900

O 2.077727000 .907690000 .23769100

O -0.57047400 - 1.536952000 .48054500

O $0.19379700-2.891644002 .54986200$

O $2.95465500-1.01241500-1.56891500$

O - $0.67888200-3.45842200-1.45141800$

H $3.41131600-0.73325600-2.37141400$

H -2.77200300 3.859724000 .55027500

H $-4.52635400-0.08113700-2.52623100$

H - $1.17600100-4.05770500-0.88672300$

H $1.449758004 .25346200-0.69816500$

H $4.781560001 .71755600-0.58175000$

$\mathrm{H}-1.017308000 .438581002 .39859500$

H -3.20809800 -0.57355900 1.51072700

H $0.411445000 .72875900-2.12870300$

H $1.25739000-1.23250700-1.87558600$

O $2.11258600-1.821304000 .85234200$

H $3.74877300-1.257944001 .38308000$

H 1.163485000 .415918002 .12673200

H $0.84052300-3.190144003 .19499500$

Intermediate

O -2.77482400 3.191532001 .10115100

O -4.64841800 - $0.38257700-1.40646600$

Si - 1.845697001 .879740000 .76994800

Si -3.25817400 -0.46025500 -0.54481100

O -2.51080800 $1.00248800-0.45425800$

Si $3.15146800-0.11504000-0.19219100$ 
Si $0.91756600-1.429658001 .55871000$ O $4.46724300-0.179319000 .86605000$ O - 1.854754000 .984393002 .14897000 O -3.67603800 -0.95875900 0.96138400 O $4.001796000 .97625900-1.25827700$ O $0.66898100-0.063722002 .49252400$ Si -0.70258300 -1.96970500 -1.00076100 Si $1.001270002 .08045800-0.57831400$ O $0.26793800-1.20086500-2.07369000$ O $1.784813003 .52193800-0.70074500$ O $0.575019001 .57102900-2.07821500$ O -2.24760800 - $1.51148700-1.30527100$ O - 0.338515002 .322412000 .35368400 O 1.924070001 .010747000 .22757100 O - $0.38111400-1.530847000 .54363600$ O $0.76030700-2.744064002 .54042500$ O $2.85455700-1.10988500-1.56359300$ O - $0.51065400-3.58531700-1.23132000$ H $3.29400100-0.71411600-2.32561100$ H -3.01523300 3.758678000 .36305600 H -4.57890700 -0.46628700 - 2.36150500 H -0.93458300 -4.17206600-0.59792600 H $1.243854004 .29171500-0.89759000$ H $4.487577001 .65706700-0.78656800$ H -0.995335000 .572010002 .38078400$ H -3.14188000 -0.54925800 1.66052800 H $0.319301000 .63205400-2.15321600$ H 1.24704400 -1.29098100 - 1.91910100 O $2.32299200-1.380437000 .78484500$ H $4.28002100-0.772466001 .59980200$ H 1.159926000 .671231002 .08969700 H $1.46045600-2.883290003 .18426100$ TS2

O -2.757503003 .179383001 .11554000$ O -4.56471300 - $0.33253900-1.58500200$ $\mathrm{Si}-1.862000001 .840808000 .80710900$ Si -3.22191000 -0.44277000 -0.65488900 O -2.48354300 $1.01767900-0.47366100$ Si $3.04198300-0.25053900-0.04387200$ Si 0.82961300 - 1.475691001 .63033300 O $4.52167700-0.161584000 .70218200$ O - 1.971936000 .914073002 .16045800 O -3.71283200 -0.99521900 0.80954200 O $4.012000000 .99489400-1.60180800$
O $0.54153900-0.153702002 .60486000$ Si -0.62609000 - $1.90643100-1.08257100$ Si $1.023360002 .07997600-0.44549900$ O $0.35395000-1.11223800-2.11945600$ O $1.817723003 .51637000-0.50656500$ O $0.631901001 .63305000-1.96968600$ O -2.17377300 - $1.46702800-1.39917200$ O -0.310949002 .228989000 .49801200$ O 2.009656001 .023512000 .31267300 O - $0.33924600-1.476309000 .47745500$ O $0.57559600-2.836510002 .50981000$ O $2.84555700-0.97219200-1.51528500$ O -0.41067300 -3.51854900 - 1.31172100 H $3.40259600-0.04350500-1.95738500$ H -2.96630000 3.753152000 .37292900 H $-4.44536700-0.38773400-2.53729100$ H $-0.86575600-4.11309000-0.70778500$ H $1.352293004 .26406100-0.89207400$ H $3.555603001 .80351100-1.85592400$ H -1.13155800 0.497092002 .44088100 H -3.23122500 -0.59812300 1.55245600 H $0.382150000 .69788800-2.10767100$ H 1.34871500 -1.16114300 -1.93623500 O $2.31717200-1.409912000 .99519300$ H $4.54716200-0.725235001 .48217400$ H 1.071572000 .608473002 .33097200 H $1.17790700-3.007655003 .23945500$ Product

O -2.89627900 3.123658000 .83799200

O $-4.33830600-0.53352100-2.02876800$ Si -2.005736001 .756672000 .66429600$ Si -3.12591100-0.56587400-0.92726300 O -2.49934100 $0.93420900-0.67118700$ Si $2.94991600-0.161433000 .37445700$ Si $0.65032000-1.407533001 .86885700$ O 4.486902000 .132236000 .90807400 O - 2.309575000 .851702002 .00429400 O $-3.77727100-1.133930000 .46731400$ O $4.329516000 .26095900-3.11445500$ O $0.08146700-0.210347002 .87536000$ Si -0.39479000 - $1.85985400-1.05341300$ Si $0.981456002 .17055200-0.30051200$ O $0.63845300-1.02181900-1.98542800$ O $1.545561003 .71318500-0.25197400$ 
O $0.732194001 .73338000-1.85796800$

O - $1.93716100-1.53749000-1.51009800$

O -0.41715400 2.08071500 0.55796700

O 2.089031001 .261305000 .46986600

O -0.27906400 - 1.398334000 .52705200

O $0.41311100-2.863541002 .58075700$

O $2.94267500-0.81885300-1.07080100$

O - $0.07211100-3.46382000-1.21815000$

H $3.83771200-0.12739500-2.35082100$

H -3.05004300 3.655308000 .05192800

H -4.08741500 - $0.58399400-2.95542800$

H $-0.58437300-4.07123800-0.67608600$

H $0.941442004 .40863600-0.52633200$

H $5.258086000 .20914000-2.87252300$

H - 1.517328000 .447125002 .41204800

H -3.43008500 -0.69394300 1.25964100

H $0.572611000 .78030400-2.01589600$

H $1.63026700-0.99669500-1.64477000$

O $2.21776600-1.129764001 .53085900$

H 4.565108000 .530251001 .77978700

H 0.648936000 .567066002 .93385100

H $0.83819400-3.019426003 .42904600$

$\mathbf{T}_{4}^{-} \rightarrow \mathbf{R}_{3}^{1-}+\mathbf{H}_{2} \mathbf{O}$

Reactant

Si -2.95214700 - 1.11157500-0.12685700

O -2.68472600 $0.47802100-0.60853200$

Si $-1.642749001 .63908200-0.14518800$

O -0.07946300 $1.18059700-0.43455700$

O - $1.63645500-1.770615000 .47732100$

Si $0.77600800-0.022133000 .29901500$

O - 1.785694002 .012760001 .45889300

O -2.02556800 2.95474200-1.04716400

O $0.12406000-0.282163001 .79459900$

O 2.336182000 .451773000 .41601300

O -3.62301700 - $1.84198500-1.46572000$

O $-4.25093600-1.099456000 .91937300$

H -1.23110800 1.457994002 .02564700

$\mathrm{H}-1.517057003 .75507900-0.88939600$

H -0.62683500 -0.91300500 1.63214400

H -3.11993000 -1.74544000 -2.27878700

H -4.00390700 -1.20530200 1.84185700

O $0.74896400-1.40944000-0.59698600$

H -0.13577100 -1.82494200 -0.41776200
Si $3.76492000-0.16021400-0.12175100$

O $3.54710200-1.71382900-0.61672600$

H $2.61673200-1.94965500-0.77424300$

O $4.244055000 .86513400-1.31384100$

H $5.119241000 .73004200-1.68786000$

O $4.92955800-0.116959001 .03815800$

H $4.90169800-0.805665001 .70868800$

TS1

Si -2.85936600 -0.99493300 -0.17840600

O $-2.858733000 .65469100-0.50248100$

Si - $1.602132001 .63516400-0.13679100$

O -0.17260200 $0.87404900-0.42270300$

O - $1.47227200-1.385903000 .50473800$

Si $0.57353200-0.334806000 .43549800$

O -1.62693300 2.136030001.44067100

O - $1.786010002 .94421700-1.11197900$

O $0.23022300-0.306202002 .06463100$

O 2.146818000 .247367000 .49599600

O -3.23463500 - $1.76564900-1.60469300$

O $-4.21800300-1.347531000 .71229400$

H - 1.058450001 .618019002 .02583600

H - $1.201586003 .68930700-0.94692300$

H -0.60057800 -0.79369000 2.17427100

H -2.60017900 -1.63415100 -2.31444800

H $-4.11857800-1.221797001 .65969600$

O $0.87001900-1.75243900-0.39037400$

H $0.03480600-2.24172100-0.43257400$

Si $3.58933700-0.07336800-0.17775700$

O $3.59453500-1.59012200-0.83030500$

H $2.69623300-1.96354800-0.86104200$

O $3.864507001 .10689700-1.29888300$

H $4.736538001 .12668600-1.70267800$

O 4.832221000 .043248000 .90477600

H $4.93951100-0.697120001 .50873700$

Intermediate

Si -2.73201200 -0.92901900-0.22396500

O $-2.804976000 .73196800-0.33345100$

Si - $1.475884001 .67871200-0.10122600$

O -0.14148800 $0.79045900-0.40859100$

O -1.30605600 - 1.333935000 .40974900

Si $0.37194000-0.562262000 .48408400$

O -1.40540800 2.263369001.44619400

O -1.66050500 $2.94358300-1.13200700$

O $0.15913200-0.396552002 .15839400$ 
O 2.000252000 .029494000 .63729400 O -3.03937400 - $1.59924000-1.70515700$ O $-4.02269000-1.480339000 .64530200$ H -0.88477300 1.703274002 .03869500 H - $1.059184003 .68477600-1.01783600$ H $-0.66880000-0.812669002 .41888700$ $\mathrm{H}-2.36467900-1.45371000-2.37413100$ $\mathrm{H}-4.03538000-1.236942001 .57491100$ O $0.93202600-1.92917000-0.35941200$ H $0.19967300-2.51381900-0.57725600$ Si $3.38508700-0.03318000-0.17973000$ O $3.57596300-1.51298100-0.90338600$ H $2.71187100-1.96623800-0.89395800$ O $3.405437001 .20077000-1.28822600$ H $4.217419001 .30649400-1.79142400$ O 4.712320000 .243403000 .77709000 H $4.95897100-0.477290001 .36349200$ TS2

Si -2.44567500 -0.76990400 0.60305900 O -2.20429600 0.859646000 .56631900 Si - $1.149772001 .75742200-0.33913100$ O $-0.228611000 .70150600-1.18020200$ O - $1.16856800-1.48828800-0.14092300$ Si $0.25466500-0.85343800-0.70551900$ O -0.22947000 2.736591000 .60031500 O -2.09878400 $2.68598600-1.31056500$ O $1.06179000-3.13504800-0.48985500$ O $1.27113900-0.506281000 .56904200$ O -3.86157300 -1.20542700-0.10291300 O -2.64439300 -1.28972900 2.14559900 H 0.698883002 .460131000 .71980800 H - $1.693731003 .47711600-1.67685800$ H $0.31573200-3.74194700-0.50754800$ H -3.93714300 -1.05721000 - 1.04983000 H -1.93701500 -1.09119500 2.76570600 O $1.00303700-1.37358800-2.06654800$ H $1.17321100-2.34447900-1.66203000$ Si 2.725632000 .228381000 .47818200 O $3.38923100-0.01925000-1.00967000$ H $2.78656300-0.44662700-1.64389900$ O 2.416841001 .829182000 .78822100 H 3.160687002 .430683000 .88708600 O $3.77128100-0.254080001 .64965700$ H $4.22391600-1.090672001 .50748800$
Product

Si 1.909507001 .202494000 .91061600 O $2.40674300-0.277584000 .36946900$ Si $1.74919300-1.31855400-0.73322000$ O $0.55200400-0.50258300-1.51287700$ O $0.782768001 .75430800-0.16260800$ Si $-0.355734000 .71039400-0.79587300$ O $1.15557100-2.67713900-0.03243300$ O $2.98484700-1.73412400-1.73111300$ O $-2.842589003 .56763600-1.36160300$ O -0.95143200 -0.02354200 0.61073100 O 3.174324002 .226785001 .07383900 O 1.275576001 .100331002 .42403700 H $0.19920800-2.678467000 .15950400$ H $2.87447400-2.54215800-2.24018100$ H $-3.217118003 .52368000-0.47775000$ H 3.789703002 .289330000 .33805700 H 0.395578000 .702208002 .42972900 O - $1.505717001 .27761900-1.70406700$ H -2.33298300 $2.72567300-1.47279100$ Si -2.26913600 -0.99958300 0.63500000 O -3.29920700 -0.51689200-0.55075900 H $-2.868793000 .15357000-1.13337600$ O $-1.63945100-2.518954000 .40843300$ H -2.21788600 -3.28166900 0.49904600 O -3.02414300 -1.03697600 2.09298900 H $-3.65015800-0.329761002 .27414200$ $\mathbf{T}_{5}^{-} \rightarrow \mathbf{R}_{3}^{2-}+\mathrm{H}_{2} \mathrm{O}$

Reactant

Si $3.41640500-1.311078000 .63241900$ O $3.676126000 .20442100-0.04620800$ Si $2.800973001 .21865000-0.96706100$ O $1.418011001 .67564500-0.17333100$ O $1.86174800-1.626431000 .77743200$ Si 0.145398000 .745539000 .26776900 O $2.368830000 .55395700-2.41546500$ O $3.757599002 .52218600-1.22959900$ O $0.07665200-0.57414900-0.73827900$ O - 1.227633001 .626880000 .13791600 O $4.30691000-1.298734002 .03868400$ O $4.26371300-2.41225100-0.28540800$ H $1.556562000 .03139400-2.37884800$ H $3.407115003 .21638400-1.79475400$ 
H $0.73185600-1.22327700-0.32367500$ H $4.11821100-0.571180002 .63748200$ H $3.70955500-2.95734800-0.85004700$ O 0.238580000 .235793001 .83531600 H $0.85455200-0.536643001 .83672400$ Si -2.73842900 1.298340000 .69563000 O -2.61109600 0.388761002 .07417600 H - 1.698362000 .247640002 .37923100 O -3.463058002 .745601000 .91414100$ H -4.42248100 2.764405000 .97223700 O -3.58198800 $0.40114000-0.38276300$ Si -3.60059200 -1.22596600 -0.70102500 O -3.66228900 -2.01856000 0.74088700 O -2.31401100 -1.62946600-1.62869200 O $-4.96261300-1.64548900-1.51087500$ H -4.94126100 - $1.55966300-2.46804900$ H -3.37302500 -1.47723000 1.49049600 H -1.43914700 -1.28569000 -1.35249000 TS1

Si $3.50919700-1.198908000 .56531400$ O $3.918586000 .19732900-0.27862900$ Si $2.828608001 .16819000-1.00940400$ O $1.505826001 .38717600-0.05024200$ O $1.91990200-1.318916000 .61218800$ Si 0.277765000 .371089000 .35517500 O $2.326144000 .57559500-2.46911700$ O $3.613329002 .58973500-1.25042600$ O $-0.07933800-0.75433400-0.83381400$ O - 1.046633001 .385557000 .23213200 O $4.30457000-1.121714002 .02357400$ O $4.29948100-2.48192100-0.13686500$ H $1.495614000 .08485200-2.44859100$ H $3.155118003 .25415500-1.77259000$ H $0.56401100-1.47410400-0.69366300$ H $4.06639300-0.374550002 .57900100$ H $3.83365100-2.88048200-0.87679400$ O $0.13066000-0.069451001 .95045800$ H $0.79055100-0.763340002 .11206300$ Si - 2.579992001 .273500000 .76108100 O -2.62944900 0.307481002 .11118000 H - 1.737945000 .060305002 .41635800 O -3.10660500 2.802200001 .03137900 H -4.05603500 2.935044001 .10316000 O -3.56458600 $0.55877400-0.34465100$
Si -3.79371500 - $1.03659500-0.71958000$ O -3.93700400 -1.87238600 0.69311000 O -2.57765000 - $1.56279900-1.68337800$ O -5.20894800 -1.26057500 - 1.52061000 H -5.18787900 -1.14185300 -2.47425700 H -3.58268600 - 1.387852001 .45521500 H - $1.67314400-1.28804400-1.42642600$ Intermediate

Si $3.29110700-1.432030000 .36466200$ O $3.91434100-0.04810900-0.30713500$ Si $3.079778001 .29931600-0.74192200$ O $1.500000001 .10493600-0.42244600$ O $1.69206700-1.271531000 .47145400$ Si $0.41421800-0.013626000 .21454200$ O $3.299377001 .65678700-2.33899900$ O 3.762415002 .531716000 .11057000 O -0.34458100 -1.09796400-0.86392100 O -0.89324600 1.126825000 .00466100 O $4.04052500-1.729460001 .80667700$ O $3.76073100-2.74861300-0.51327500$ H $2.737147001 .18515400-2.96040400$ H $3.489828003 .42266300-0.12608900$ H $0.18223300-1.90187600-0.92719200$ H $3.92695600-1.057811002 .48469400$ H $3.33730700-2.85369700-1.36957300$ O $0.11053000-0.054189001 .89314000$ H $0.67790900-0.701106002 .32345300$ Si -2.37019200 1.337444000 .59577400 O -2.53950500 0.520905002 .04056800 H - 1.652961000 .215337002 .32154300 O -2.63516800 2.958763000 .75284500 H -3.54492900 3.232074000 .90024700 O $-3.564881000 .73690500-0.37935700$ Si -4.00470800 -0.83899500-0.60823800 O -4.18258700 - 1.534473000 .87797500 O -2.90944600 -1.59156600-1.56565500 O -5.47733300 - $0.95152700-1.33166100$ H -5.48484500 -0.91085700 -2.29196000 H -3.71062900 -1.03532600 1.56579800 H -1.96677900 -1.39931800 -1.35152100 TS2

Si $3.41540700-0.12674400-0.62005300$ O $2.71449000-1.57284900-0.24372500$ Si $1.24506400-1.887346000 .43594500$ 
O $0.54423100-0.486149000 .84338500$ O $2.392746001 .05722600-0.13637800$ Si 0.875855001 .143019000 .50529300 O $1.41356600-2.935550001 .68722200$ O $0.24321900-2.63536400-0.65006400$ O 0.568616001 .823492001 .97035900 O -0.27154200 $1.46734800-0.65438200$ O $3.75729800-0.02520000-2.22002400$ O 4.894109000 .017279000 .07123100 H $2.08093800-2.729863002 .34850200$ H $0.49323100-3.51862200-0.94070500$ H 0.819672002 .795333001 .62666600 H $3.01273700-0.04714800-2.82787500$ H $4.93265400-0.013083001 .03126400$ O 1.301274003 .522924000 .49398000 H 0.598603003 .979782000 .02157900 Si - $1.879088001 .59496600-0.40179100$ O -2.16849600 1.737003001 .22815700 H - 1.359199001 .879824001 .75782800 O $-2.382008002 .89318700-1.26974300$ H -3.32241100 2.95696100 -1.45925100 O -2.70049600 $0.26914300-0.90329800$ Si -3.30498500 - $1.09515200-0.19421200$ O -3.23463600 -0.90670900 1.43819600 O - $2.51416100-2.42322600-0.72262200$ O $-4.88895600-1.29675400-0.59338400$ H -5.07052300 - $1.68551700-1.45369000$ H -2.93194000 -0.02454300 1.71015200 H - $1.53935100-2.44193400-0.67828700$ Product

Si -3.35452700 -0.12047100 0.65804200 O -2.66553800 - 1.593272000 .35306500 Si - $1.30331300-2.01185700-0.47165600$ O $-0.62312000-0.65289000-1.05714600$ O -2.34357900 1.021573000 .05803900 Si $-0.789382000 .93057600-0.52823100$ O - $1.64287300-3.12180300-1.62848800$ O $-0.18002000-2.702614000 .52582600$ O -0.37315700 $1.99292400-1.61795700$ O 0.185289001 .035147000 .83247200 O -3.64684300 0.058596002 .26094100 O $-4.85231900-0.004390000 .00352200$ H -2.39821400 -2.95254400 -2.19925600 H $-0.38197000-3.567206000 .89784500$
H -0.85578600 $3.60820600-1.59683500$ H -2.89607700 -0.02995500 2.85505800 H -4.90757700 $0.02516500-0.95579100$ O -1.11434000 4.56428300 -1.60004900 H $-0.790268004 .91715300-0.76700500$ Si 1.773958001 .426309000 .80742500 O $2.122569002 .06926200-0.67780300$ H $1.278332002 .20408700-1.19386000$ O 2.036230002 .448412002 .06454300 H 2.942509002 .544604002 .37053000 O 2.727880000 .101656001 .00625900 Si $3.33879100-1.02426900-0.03657900$ O $3.23757200-0.42199200-1.56456800$ O $2.57359100-2.458931000 .13946700$ O $4.93353600-1.296893000 .26813300$ H $5.13528700-1.896689000 .99187300$ H $2.951929000 .50675900-1.59110700$ H $1.60542000-2.476169000 .25248400$

$$
\mathbf{T}_{\mathbf{5}}^{-} \rightarrow \mathbf{R}^{1-}+\mathbf{H}_{2} \mathbf{O}
$$

Reactant

Si -2.10771000 - 2.31993600-0.21227500

O $-0.54032900-2.35208200-0.45641200$

O -2.85005400 -0.87101800-0.56840100

O $-2.95676700-3.44579900-1.09626400$

Si $1.377584000 .21630300-0.35500700$

Si -3.072336000 .518312000 .26348700$

H -2.40059600 -4.02364400 - 1.62547200

O $0.849753001 .74212000-0.04964200$

O -1.801599001.507895000.06195000

O $3.011026000 .36255100-0.48242800$

O $1.05064500-0.811994000 .89433000$

O $-4.353258001 .36238100-0.33145000$

O -3.28664500 0.168520001 .85829700

Si $-0.576732002 .54404300-0.13066300$ H $0.41083400-1.516393000 .56429300$

H $-5.214877000 .94147500-0.26252800$

H -3.06831500 -0.76090800 2.05073800

O $-0.809494003 .25898400-1.59127100$

O -0.568188003 .727416001 .01233800$

H -0.17115400 $3.92731100-1.85656200$

H - 0.199098003 .497881001 .87022500

O -2.54622700 -2.49921600 1.40624900

H -2.00124600 -3.10263400 1.91902400 
O $0.70607500-0.37528900-1.72691500$ H $0.15627600-1.17090000-1.49438600$ Si $4.26959800-0.447917000 .19205300$ O 5.412348000 .579707000 .78328200 H 5.996967000 .995023000 .14298700 O $3.74862100-1.313373001 .48728200$ H 2.77637000 -1.37298900 1.52617100 O $4.88141300-1.37521200-1.02654100$ H $5.67064300-1.89023000-0.83603200$ $\mathrm{TS} 1$

Si -2.26963000 $1.70967200-0.13614100$ O -0.98412800 2.382268000 .49946500 O -2.78072400 0.300519000 .61469600 O -3.65648400 2.63058800-0.12003300 Si 1.116693001 .088021000 .62960200 Si -2.97922500 -1.20967500 0.02285900 H $-3.518107003 .56685300-0.28701400$ O $0.20431100-0.06843200-0.14101500$ O - $1.55740200-2.046693000 .09032900$ O 2.594265000 .325306000 .79825800 O $1.479235002 .33986100-0.39243700$ O -3.99713800 -2.10796000 0.94373200 O -3.53176900 - $1.14978900-1.52751800$ Si $0.02445500-1.672508000 .13336600$ H $0.663637002 .85790500-0.50034700$ H -4.91646300 -1.82865000 0.97518600 H -3.17537600 -0.37284700 - 1.99182000 O $0.63123300-2.133775001 .58519900$ O $0.86757700-2.51247000-1.02198200$ H $1.51430600-1.816849001 .80541400$ H $0.54541600-2.45028300-1.92756200$ O - $2.059852001 .26797200-1.75586600$ H - $1.148542001 .01580400-1.94370600$ O 0.715843001 .389701002 .19382100 H -0.13352200 1.864279002 .14480200 Si $3.75800600-0.06988100-0.29647900$ O $3.58711800-1.62859500-0.79016000$ H $2.69105900-1.96099600-0.97080400$ O $3.700819000 .99732100-1.55000300$ H $3.021202001 .68280100-1.44798500$ O 5.262082000 .050284000 .36009900 H $5.55232400-0.685664000 .90625600$ Intermediate Si -2.07157800 $1.68737600-0.19451000$
O -0.64363900 2.17559400 0.36926600 O - 2.705108000 .379502000 .60573300 O -3.23015900 $2.84901900-0.01595100$ Si 0.874798001 .200201000 .53609300 Si -2.98709300 - 1.159306000 .10570700 H -2.97308100 $3.74136000-0.26432600$ O $0.11398700-0.06919100-0.32879800$ O - $1.59936000-2.042118000 .14518400$ O 2.350145000 .239044000 .72776800 O $1.701558002 .35435900-0.39069300$ O -3.99849900 - 1.949226001 .12363000 O $-3.62705100-1.13686700-1.41206500$ Si $-0.01064300-1.652250000 .04340200$ H $1.102893003 .04700800-0.68682100$ H $-4.90882000-1.642119001 .16078400$ H -3.29826800 -0.39359900 - 1.94094700 O $0.73540200-2.023326001 .45716900$ O $0.72331300-2.57503300-1.12419500$ H $1.47294900-1.414222001 .62757800$ H $0.34131100-2.54652100-2.00738900$ O $-2.025920001 .28501200-1.81801800$ $\mathrm{H}-1.177051000 .90325000-2.07242400$ O 0.747958001 .270782002 .21083300 H - 0.046208001 .754718002 .45671200 Si $3.61056200-0.15539600-0.22164400$ O $3.44982700-1.67607700-0.85372400$ H $2.55594500-1.98889400-1.06885000$ O $3.809489000 .97560100-1.40842200$ H $3.145958001 .68168500-1.29151200$ O $5.04309200-0.189117000 .60980100$ H $5.19945000-0.975090001 .14039500$ TS2

O -2.63657300 $0.55315400-0.01998700$ O $0.28626300-0.68545900-1.59236300$ Si $0.891360000 .79930900-1.04536100$ Si -0.31624500 -2.06751000-1.02097900 Si - $1.788184001 .96555000-0.14989400$ Si -2.45249300 -0.79933900 0.88063800 O $-3.88982000-1.525313001 .17971700$ H -4.21258000 -2.15330400 0.52691200 O -1.82445100 -0.24869600 2.30290500 O -1.06644600 -2.94083300-2.19469100 O - 1.436832002 .495980001 .36828900 O $0.93614000-2.93465900-0.35179700$ 
O -2.66156200 $3.09087300-0.95759100$ O $2.076302001 .06006700-2.15558500$ H - $1.52345900-0.883632002 .95903000$ H -3.46523300 $3.40650100-0.53473100$ H -1.39259300 1.788456002 .02805400 O - $1.45389700-1.850757000 .13756700$ O -0.46832000 $1.72405700-1.06495400$ H $0.77759500-3.87601600-0.22643700$ H $2.355370001 .97528100-1.75831500$ O $2.001532002 .90313900-0.63573400$ H 2.597773002 .857516000 .11809000 O 1.367178000 .387737000 .49031800 Si $2.79683600-0.224657001 .02354800$ O $3.89235000-0.19793100-0.20871800$ O 3.447358000 .675244002 .23777400 O $2.55430900-1.740155001 .61095300$ H $3.549884000 .14848500-1.04897600$ H 3.058373000 .570261003 .11053800 H $1.99576100-2.283471001 .02485100$ H -0.56699700 -3.10858800 -2.99917200 Product

O 2.377438001 .013465000 .58639600 O -0.03314700 - $0.72476800-0.79834400$ Si -0.79277300 $0.61607600-0.13426900$ Si $0.54444300-2.05005500-0.04855900$ Si $1.773786002 .00268500-0.59241700$ Si $2.93919900-0.525465000 .67397100$ O $4.12910600-0.629829001 .78881400$ H $3.97297300-0.239304002 .65357000$ O $3.56893800-0.88693200-0.81387000$ O - $0.60012700-2.917261000 .73514800$ O $2.463282001 .57429700-2.02410400$ O $1.28761100-2.93417100-1.23000100$ O $2.073998003 .57864100-0.25773800$ O - 1.047385000 .475600001 .42403100 H $3.28308900-1.72797700-1.19555700$ H $2.982495003 .87862000-0.35312800$ H $2.923315000 .72174800-1.99784300$ O $1.72344500-1.577258001 .01607900$ O $0.140919001 .89289500-0.62668100$ H $1.37301800-3.88204500-1.09441300$ O -3.005633002.091150002.44627700 $\mathrm{H}-3.720248001 .834126001 .84812100$ O -2.24196700 $0.70001900-0.96507500$
Si -3.53564800 -0.16064900-0.40863400 O -3.02649300 - 1.161928000 .80509500 O -4.67564700 0.841997000 .24580100 O $-4.25675600-0.99940600-1.62143200$ H -2.33617400 -0.66147700 1.32595900 H -5.09410000 $1.47596400-0.34474600$ H -3.67955100 -1.46686900 -2.23240700 H - $1.49867700-2.549178000 .71582100$ H -2.23287600 1.567984002 .13017200

$$
\mathbf{T}_{6}^{-} \rightarrow \mathbf{R}_{3}^{3-}+\mathbf{H}_{2} \mathbf{O}
$$

Reactant

Si -3.75067400 1.18868500 1.15009600

O -4.22094500 -0.06481900 0.13385100

Si -3.56295200 -0.84108100-1.13483300

O -2.18492900 -1.64197800 -0.67502900

O -2.16345700 1.298098001 .23826800

Si -0.78885700 -0.98435900 -0.11667600 O -3.18029800 $0.17981400-2.37559700$ O $-4.69602500-1.91169300-1.64050900$ O $-0.684929000 .55887100-0.70923800$ O $0.46491500-1.90646500-0.61658600$ O -4.55772300 0.902025002 .57670100 O -4.506789002 .572492000 .61556300$ H -2.31383400 $0.59701700-2.27646800$ H $-4.48396200-2.43962900-2.41541800$ H - $1.217074001 .11117100-0.04294700$ H -4.42649300 0.027394002 .95270200 H -3.93053200 3.169587000 .13111000 O $-0.72944100-0.953681001 .53283900$ H -1.23772000 -0.15759400 1.82247000 Si $1.87582800-2.357281000 .08669900$ O $1.72430400-2.301519001 .73246700$ H $0.90701700-1.868972002 .03858500$ O $2.20844400-3.85454300-0.47452700$ H $3.12414700-4.14718200-0.45682400$ O $3.09323500-1.33224300-0.32116000$ Si $3.77043800-0.081170000 .50928300$ O $5.147862000 .41310000-0.24238800$ H $5.84673500-0.22678000-0.40558500$ O $4.06244600-0.531877002 .05758600$ O 2.787752001 .223688000 .46450600 H $3.41310600-1.164831002 .39933100$ Si $2.457594002 .36746600-0.68377200$ 
O $1.457429001 .78269200-1.83208300$ H $0.653913001 .33342800-1.49230500$ O $3.872392002 .84262000-1.38060500$ O 1.713244003 .647477000 .03242400 H 2.187933004 .088103000 .74314000 H $4.597590002 .21019100-1.28083400$ TS1

Si -3.98881800 0.99509100 1.13776600 O $-4.566529000 .06351800-0.13848600$ Si -3.60946000 -0.61108700 - 1.27631700 O -2.25550100 -1.25418300 -0.59221000 O -2.39497900 0.993261001 .09486800 Si -0.92262300 -0.53416400 0.05822400 O -3.15127500 0.46931400-2.44229200 O -4.52511100 - $1.78435100-1.96865000$ O $-0.570661000 .93517800-0.65863000$ O $0.31067500-1.47080500-0.56874400$ O -4.70796700 0.403368002 .51613900 O -4.70007900 2.494944001 .04782700 H -2.27916900 $0.86183800-2.31332900$ $\mathrm{H}-4.16149800-2.21119100-2.74956300$ H - $1.130221001 .58770300-0.20000800$ H $-4.52992100-0.522395002 .70285300$ H -4.225670003 .127257000 .50135000$ O -0.65241000 -0.77857700 1.67823700 H -1.25774100 -0.19682500 2.16472400 Si $1.62221100-2.25216800-0.02464700$ O $1.57773300-2.386366001 .62809300$ H $0.81109600-1.917750002 .00665600$ O $1.66589200-3.70853900-0.77742900$ H $2.50957800-4.16897400-0.79618700$ O $3.01557800-1.44399100-0.38111200$ Si $3.86019900-0.360376000 .52208600$ O $5.29467300-0.01147800-0.20800100$ H $5.89292300-0.73178000-0.42640500$ O $4.08093200-0.945865002 .03691400$ O 3.072779001 .074173000 .56918200 H $3.35678300-1.528502002 .31617600$ Si $2.856699002 .26584500-0.55738100$ O $1.776494001 .82333300-1.69733400$ H $0.915945001 .48921800-1.36495000$ O $4.304980002 .57950600-1.27833500$ O 2.283749003 .613164000 .19147700 H 2.817669003 .976019000 .90434700
H $4.953537001 .86658700-1.19402900$ Intermediate

Si -3.84553100 1.48547800 0.69205000 O $-4.572970000 .25549900-0.15314600$ Si -3.82693300 - $1.05221400-0.81479700$ O $-2.21478300-0.88635500-0.71158200$ O -2.27112300 1.164839000 .80607100 Si - 1.053180000 .019112000 .11296600 O $-4.26833200-1.26482300-2.39042800$ O $-4.40511100-2.352969000 .01446600$ O -0.35437600 $1.34261100-0.69977500$ O $0.23024400-0.98443200-0.50948800$ O -4.574546001 .680487002 .16047800$ O $-4.198553002 .92786200-0.03267600$ H -3.79300400 -0.73821800 -3.03955300 H -4.15237400 -3.22029000 -0.31445600 H -0.84019800 2.15010800 -0.50412900 H -4.368014001 .013571002 .82104500$ H -3.92650800 $3.01385800-0.95060000$ O $-0.65592900-0.431105001 .70775400$ H -1.21689200 0.021121002 .34466800 Si $1.30715000-2.07247600-0.04637700$ O $1.28818100-2.281141001 .60621500$ H $0.58841900-1.701260001 .97305800$ O $1.02722100-3.48555400-0.85082900$ H $1.71854000-4.15191100-0.80563100$ O $2.86514200-1.61632000-0.40217600$ Si $3.85729500-0.680130000 .50996600$ O $5.33883500-0.55513100-0.20462100$ H $5.80556000-1.36229400-0.43971400$ O $3.97136300-1.295059002 .02690500$ O 3.308181000 .863410000 .56364500 H $3.15092600-1.748681002 .28406600$ Si $3.239148002 .04802800-0.58931400$ O $2.083483001 .75519200-1.69686400$ H $1.180439001 .55101900-1.34957400$ O $4.705904002 .12939300-1.34084700$ O 2.899014003 .483745000 .14292100 H 3.502410003 .770938000 .83437100 H $5.244636001 .33167900-1.24463700$ TS2

Si $3.64492800-0.60728800-0.91322500$ O $2.98368100-1.795646000 .01875300$ Si $1.73582200-1.715471001 .09287100$ 
O $1.17419700-0.192455001 .12281800$ O $2.914400000 .80449900-0.52025100$ Si 1.592335001 .257054000 .36008700 O $2.19487600-2.220666002 .58963400$ O $0.60994700-2.794953000 .56424200$ O 1.681734002 .260247001 .66249900 O $0.266434001 .50807800-0.61577100$ O $3.49963000-0.96533200-2.50788700$ O $5.26916200-0.51040200-0.71448600$ H $2.71617800-1.604719003 .11290100$ H - $0.24104800-2.782774001 .04020700$ H 1.969681003 .089284001 .08776000 H $2.61553000-1.16205200-2.83052100$ H $5.58567900-0.187330000 .13377800$ O $2.283241003 .48916100-0.27840700$ H $1.564492003 .96504300-0.70800600$ Si - $1.027260002 .43512000-0.27170500$ O -1.160319002.633213001.37059400 H - 0.293525002 .653052001 .81711300 O $-0.855456003 .83941200-1.10303900$ H - $1.570582004 .48018900-1.04919100$ O -2.42369800 $1.70444800-0.73116400$ Si -3.40567300 $0.60683900-0.02152300$ O -2.90103100 0.32240800 1.53103200 O -3.34803300 - $0.84436900-0.77374000$ O $-4.913116001 .23151400-0.13935400$ H -5.65417800 0.656493000 .07233900 H -2.35565000 1.031610001 .90904900 Si -2.55972900 -2.27235000-0.55837200 O $-3.55228300-3.50227200-0.99527100$ H $-4.44316700-3.49656700-0.63314400$ O $-1.24580200-2.39187400-1.52412100$ H - $0.40283300-2.48553700-1.04836600$ O -2.09756100 -2.38093800 1.03437500 H -2.25652300 -1.56390500 1.53855800 Product

Si $3.76025300-0.08816700-0.30956900$ O $2.94979700-1.38985000-0.94236500$ Si $1.35142100-1.74476000-0.97048200$ O $0.53917000-0.62416300-0.11992200$ O 2.640867000 .902292000 .36313100 Si 0.986276000 .877437000 .46616200 O $1.05193400-3.24426500-0.35413700$ O $0.68922500-1.69679000-2.47830500$
O 0.352326001 .135697001 .89762100 O $0.428143001 .97632200-0.64822400$ O $4.650318000 .65067100-1.46692700$ O $4.88972400-0.559819000 .77704800$ H $1.67133700-3.589309000 .29730700$ H $0.88455200-2.41281100-3.09115100$ H 1.172942001 .678741003 .28776200 H $4.189326000 .95462700-2.25399800$ H $4.57418200-0.909562001 .61520800$ O 1.625125001 .982031004 .11098300 H 1.635767002 .941408004 .05711500 Si - $1.111738002 .56645700-0.64837200$ O -1.556853002 .865218000 .90277300$ H - 0.998337002 .332300001 .51338700 O -1.20717800 $3.98258700-1.47195200$ H -1.39456300 $3.92533800-2.41293300$ O -2.08701900 $1.47783200-1.39541000$ Si -2.71074400-0.03086700-1.21870800 O -2.07622800 -1.04917500 -2.33301200 O $-4.338128000 .01092400-1.46318100$ H $-4.640404000 .30142500-2.32818400$ H - $1.11363900-1.17333900-2.38750500$ O $-2.49721700-0.512995000 .31426600$ Si -2.15486900 - 1.553745001 .53589200 O $-1.22878100-0.819447002 .65124500$ O $-1.55542800-2.973288000 .95143900$ O $-3.54901500-1.959271002 .32527400$ H $-0.62653400-0.075709002 .36321800$ $\mathrm{H}-0.68028300-3.001389000 .53654400$ $\mathrm{H}-4.12896600-2.576784001 .87153400$ $\mathbf{T}_{6}^{\prime-} \rightarrow \mathbf{R}_{\mathbf{4}}^{2-}+\mathbf{H}_{\mathbf{2}} \mathbf{O}$

Reactant

Si -3.68542700 - $1.20646000-0.07858400$ O $-2.86854500-2.33885100-0.89991700$ O -3.44834800 $0.30307900-0.70696700$ O $-5.30808400-1.45454800-0.19962400$ Si $0.14127400-1.426786000 .60925900$ Si -3.10581400 $1.77985600-0.07641600$ H -5.63759400 -2.31333500 0.07966900 O - 0.244095000 .188212000 .59574700

O - $1.584674002 .21800900-0.51019500$ O $1.80893400-1.494474000 .78047900$ O -0.58220200 -1.89810100 2.03464200 O $-4.039383002 .96034800-0.72594100$ 
O -3.29017200 1.728886001 .55737100

Si -0.14203000 $1.45341100-0.43502800$

H $-4.977517002 .93820600-0.51707900$

H -3.26648300 0.822990001 .90967700

O $0.249331001 .02463700-1.96717500$

O 0.990137002 .505843000 .15736800

H $0.946902000 .35732500-2.08027500$

H 1.409534002 .210661000 .98221900

O -3.27504600 - 1.132484001 .53113700

H -2.39958600 -1.47086800 1.79666900

O $-0.31361500-2.20982600-0.69461700$

Si $2.81181000-1.58713200-0.53594100$

O $3.57133800-3.03435800-0.64565500$

H $4.20431900-3.261981000 .04173600$

O $1.90192800-1.36681700-1.89212100$

H $1.01482900-1.79722200-1.74876700$

O $3.91333700-0.38373400-0.41089900$

Si 4.059817001 .066245000 .34455100

O 2.988454001 .111636001 .61300100

H 2.610707000 .254568001 .85608200

O $3.719771002 .34009300-0.62784900$

H $2.779775002 .58783400-0.64489000$

O 5.633014001 .145573000 .79338900

H 5.968966002 .000586001 .07706300

H $-0.49056000-2.828505002 .26454500$

H - $1.85477400-2.31315100-0.86382000$ TS1

Si -3.48329800 - 1.233564000 .25386900

O -2.34045100 - $2.10319000-0.40142300$

O $-3.778036000 .25681400-0.44744600$

O $-5.01063000-1.902747000 .22327100$

Si $0.05462900-1.622586000 .28872100$

Si -3.15290400 $1.73892100-0.16748300$

H -5.02128200 -2.85952300 0.31258900

O $-0.38120500-0.055245000 .07729300$

O -1.75328900 $1.95703400-1.01207700$

O $1.71486400-1.538792000 .56973700$

O - $0.54006500-2.175633001 .73050900$

O -4.12241100 $2.92791700-0.75402100$

O -2.89627800 1.917486001 .45078900

Si $-0.268034001 .31131300-0.80575300$

H -5.00624200 $2.98731200-0.38077300$

H -2.90961700 1.047410001 .89526700

O $0.354932001 .06365200-2.29960500$
O 0.691820002 .365498000 .03700900

H $1.133043000 .48163400-2.33398200$

H 1.000043002 .029801000 .89539800

O $-3.10608700-0.844400001 .85555400$

H -2.27208900 -1.27660100 2.10372500

O $0.01769500-2.56972300-1.06036600$

Si $2.90123700-1.42563700-0.56246700$

O $3.77932700-2.80128800-0.69038100$

H $4.13893000-3.181407000 .11653800$

O $2.23957100-1.11091200-2.04764100$

H $1.53386900-1.74290300-2.26337300$

O $3.85842000-0.15838000-0.18059200$

Si 3.785653001 .235485000 .68064500

O 2.533546001 .122637001 .76790000

H 2.230582000 .223647001 .95249100

O $3.502007002 .55526300-0.24577700$

H $2.559977002 .71475400-0.42756100$

O 5.263403001 .375023001 .37316200

H 5.483409002 .218444001 .77905500

H $0.13513500-2.295485002 .40658200$

H -0.94479900 -2.65026300 -1.24858100

Intermediate

O $1.66513600-1.61594400-0.23004400$ O 0.509432001 .115120000 .77061100 Si 1.988719001 .478357001 .38119600

Si $-0.203345001 .54681600-0.72200600$ Si $3.17811400-1.017455000 .01082500$

Si $0.52646900-1.36706200-1.39761400$

O $-0.37597200-2.75124500-1.51749900$

H $0.09374800-3.58837200-1.58467400$

O $1.34383100-1.04233800-2.80560500$

O -1.85610900 $1.83813600-0.61819900$

O $3.94750600-0.90857700-1.43984000$

O $0.788546001 .53290700-2.10682000$

O $4.03302800-1.967590001 .03937700$

O 2.372526003 .024851001 .00942800

O 1.998171001 .329570003 .01925600

H $4.29551100-2.833628000 .71434900$

H $3.33880500-0.95691100-2.19362000$

O - $0.53667800-0.20244700-1.04796200$

O 3.097707000 .438209000 .74896600

H 1.669152003 .389834000 .42561500

Si -3.12767300 $0.80628600-0.56004700$

O $-3.08304200-0.111630000 .79209600$ 
H 2.127408000 .445313003 .37430400

Si -2.34088600 -1.40126600 1.48957200

O -3.25496500 -1.91730200 2.76069900

H -3.42656500 -1.26911500 3.44916000

O -0.86034700 -0.96570000 2.04478700

O -2.32736200 - 2.71223300 0.50590500

H - $1.67002300-2.74537400-0.21139200$

H - $0.37569100-0.275288001 .55370500$

O -3.23540000 - $0.10487400-1.93301500$

O -4.52672500 $1.66156300-0.41173600$

H -4.69692700 2.32841600 -1.08253900

O $0.161687003 .26877600-0.54666500$

H - $0.554098003 .74497600-0.11688500$

H -2.37051000 -0.47750000 -2.15199800

H $1.137018002 .41104100-2.29157300$

H $1.24483500-0.08685500-2.98131200$ TS2

O $1.53360600-1.74774700-0.69313200$ O 0.481512001 .251923001 .07734700 Si 2.107149001 .305794001 .34028500 Si - $0.351939001 .65409400-0.33305200$ Si $2.96920000-1.30841600-0.01503400$ Si $0.52107200-0.93305300-1.70397500$ O -0.46243200 - 2.04638400 -2.42543200 H - $0.06255200-2.81796800-2.83851100$ O $1.45519800-0.02540100-2.71668000$ O - $1.987880001 .85058400-0.08974300$ O $3.93819900-0.70390400-1.20023700$ O $0.600288002 .25363700-1.55190100$ O $3.68766800-2.572092000 .73448000$ O 2.816360002 .336802000 .27918800 O 2.454557001 .827497002 .85273600 H $3.97371800-3.306472000 .18381000$ H $3.44167800-0.33468200-1.94662800$ O - $0.529753000 .01629400-0.91166400$ O $2.70268200-0.220624001 .17860900$ H $2.311081002 .43186000-0.54889700$ Si -3.19560000 $0.74590100-0.25442200$ O $-3.02335200-0.475256000 .81477400$ H 2.61743400 1.155065003 .51998100 Si -2.10513100 -1.77050400 1.23294300 O -2.91190100 -2.68709700 2.33749200 H -2.99982900 -2.30873900 3.21649200 O $-0.70936200-1.265898001 .93692600$
O - $1.92679400-2.82349300-0.01232800$

H - $1.53117800-2.53849700-0.84974800$ H $-0.29340800-0.455788001 .59935300$ O $-3.316403000 .19818700-1.80621100$ O -4.62873000 1.443499000 .14425600 H -4.87002100 2.24552800 -0.32732600 O -0.166110003 .635836000 .05251600$ H -0.99579100 $4.11231100-0.05888200$ H -2.47836500 -0.12136400-2.16136000 H $0.350642003 .32608900-0.97584600$ H $1.251253000 .92833900-2.55485200$ Product

O $1.74564700-1.35492800-1.16090500$ O 0.368168000 .523252001 .54510100 Si 1.965767000 .619057001 .98629200 Si -0.324007001 .338049000 .25300600$ Si $3.13740200-1.09992300-0.31312300$ Si $0.69958500-0.34512900-1.92331500$ O -0.09795500 - $1.18609000-3.08318100$ H $0.39157000-1.82621800-3.60876500$ O $1.538254000 .98164100-2.42935800$ O - 1.882870001 .665275000 .66629600 O $4.06507600-0.02338500-1.14111900$ O $0.614935002 .50867300-0.25806700$ O $3.94670200-2.49930100-0.06914100$ O 2.524960002 .111598001 .62521300 O 2.154477000 .379874003 .59483200 H $4.31452100-2.93682700-0.84232900$ H $3.562876000 .54733100-1.74088000$ O -0.49822300 $0.14387500-0.91706500$ O $2.77225100-0.570798001 .18880800$ H 1.977487002 .504584000 .90263500 Si -3.22500200 0.80746100 0.23735400 O -3.03320900 -0.76060400 0.63551600 H $2.22291600-0.529623003 .89917200$ Si -2.22898900 -2.17511100 0.40935700 O -3.13698000 -3.42424400 0.97539700 H -3.31377200 -3.42916900 1.91999200 O $-0.81520400-2.136529001 .24575800$ O - $2.08100900-2.56580400-1.17813300$ H -1.52418400 -2.03198900 -1.75992500 H $-0.38480600-1.277094001 .37835400$ O -3.54082800 $1.00212500-1.36779300$ O -4.524938001 .323843001 .08735200$ 
H -4.77899300 2.24654500 0.99677700

O $-0.241400004 .73442000-1.52681600$

H - $0.420138005 .38870200-0.84611900$

H $-2.805322000 .82253900-1.96333700$

H $0.057194003 .92789000-1.04475300$

H $1.331234001 .74848600-1.84636500$

$\mathbf{T}_{6}^{-} \rightarrow \mathbf{R}_{5}^{1-}+\mathbf{H}_{2} \mathbf{O}$

Reactant

Si - 1.766106000 .984798000 .59190000

O 1.083931002 .740285001 .10212700

O -0.88741600 -0.29111400 0.06745000

Si - $0.49583300-1.27309800-1.18276200$

O $0.52906800-2.43002600-0.61692300$

Si $1.92561500-2.511185000 .21951200$

O $2.94546700-1.32603800-0.24713100$

Si 3.609551000 .055014000 .36702300

O $2.950791001 .34725400-0.34039400$

Si $1.797832002 .54773000-0.29540100$

O $1.72099100-2.249967001 .83793600$

O $2.57540100-4.002291000 .00958800$

H $2.42332300-4.43146200-0.83735800$

O $5.204664000 .12628400-0.03296500$

O 3.437367000 .030232002 .00556200

O $2.568706003 .90885000-0.88356800$

H $3.071424004 .39803000-0.22672100$

O $0.780527002 .11717400-1.56172500$

H $-0.158363002 .30419200-1.38385400$

O - 1.070258001 .722108001 .84303200

O -3.25341100 0.458885001 .05367300

O - $1.79933000-2.08947600-1.74044400$

H -2.66412700 -1.67390900 - 1.56912400

O $0.22164700-0.42024400-2.37962600$

H $0.559226000 .46679400-2.12634300$

H $5.76754900-0.563800000 .32933000$

H -0.156784002 .152595001 .57647000$

O - $1.902625001 .96317400-0.75434300$

H $2.81047900-0.634837002 .32802600$

H $1.14240700-2.833249002 .33695300$

H -2.42664700 $2.76391600-0.65090600$

Si -4.60880400 -0.15227900 0.39267600

O -4.19456300 -0.82279200-1.06714700

H -4.88147300 - $0.86737500-1.74000600$

O -5.13688600 -1.25046100 1.49336400
H -5.98354300 -1.67575600 1.32908900

O -5.740479000 .983334000 .03306300$

H -6.24379500 1.359758000 .76090900

TS1

Si - 1.694961000 .730942000 .88969100

O 0.489660001 .852484000 .94991900

O $-0.79326200-0.544810000 .39239000$

Si $-0.40110900-1.56668700-0.81267400$

O $0.87416300-2.48049200-0.30761700$

Si $2.34678800-2.201606000 .33267300$

O $3.04091700-0.92333600-0.40803600$

Si $3.665211000 .55552100-0.02908600$

O $2.730360001 .73182900-0.63441400$

Si $1.210599002 .36386000-0.35942200$

O $2.30488000-1.766998001 .92554200$

O $3.24240900-3.569909000 .19659800$

H $3.06974900-4.13357800-0.56301900$

O $5.115773000 .76722300-0.77620000$

O 3.862195000 .636925001 .60537100

O $1.400001004 .02154600-0.47214300$

H 1.481739004 .463017000 .37759100

O $0.389519001 .94224000-1.76704000$

H -0.56140300 2.04561200 -1.57757500

O -1.44061300 1.22419400 2.43494600

O -3.224273000 .035952001 .06812700$

O - $1.58734500-2.65594700-1.10968500$

H -2.49350600 -2.29798900 -1.07991900

O $-0.02228500-0.74804500-2.18014800$

H $0.252205000 .18766300-2.07292000$

H $5.823541000 .17282400-0.51180800$

H -0.566387001 .657327002 .39724900$

O -1.96044200 $1.85955400-0.31309200$

H $3.34660900-0.020897002 .09552800$

H $1.98065700-2.398387002 .57365800$

H -2.87161000 $1.86213900-0.64659200$

Si -4.49171600-0.21695800 0.10135900

O $-4.12257100-1.48451800-0.90465900$

H -4.67408500 -1.60217400 -1.68509400

O $-5.78536300-0.506626001 .06590000$

H -6.59337500 -0.83378200 0.66024200

O $-4.726345001 .08961900-0.89809100$

H -5.38049400 $1.74013100-0.62426200$

Intermediate

Si -1.37186700 0.93569600 0.76574500 
O 0.073347002 .003363000 .71880500

O -0.44366100 -0.40223200 0.29768900

Si - $0.31729500-1.50908700-0.87879700$

O $1.02202500-2.43243400-0.57310000$

Si $2.44633700-2.263101000 .19136000$

O $3.22761900-0.91591300-0.30892800$

Si 3.437152000 .625627000 .22982600

O $2.440843001 .62680500-0.56881600$

Si $0.992902002 .40766700-0.53768000$

O $2.28982700-2.045117001 .82319300$

O $3.35306000-3.60388800-0.07356500$

H $3.23923600-4.04900600-0.91820200$

O $4.941726001 .15836300-0.15836200$

O 3.211074000 .655856001 .85900500

O $1.299736004 .03370500-0.59997900$

H 1.667897004 .427383000 .19611800

O $0.247228002 .02085200-1.97384000$

H - $0.716633002 .08131300-1.81121600$

O - 1.504009001 .254143002 .41536400

O -2.84618900 -0.04817500 0.89700600

O - $1.51478300-2.62248600-0.95739300$

H -2.43849400 -2.31508600-0.88869000

O $-0.15271500-0.77153300-2.34540000$

H $0.127941000 .16127700-2.30985800$

H 5.681597000 .695391000 .24499600

H -0.782043001 .833325002 .67834100$

O -2.02990600 $1.87520400-0.50273700$

H $2.80104600-0.149309002 .21199600$

H $1.73627000-2.654483002 .31966600$

H -2.92515100 $1.62605400-0.77263200$

Si -4.26352500 -0.37300500 0.25318900

O -4.12009000 -1.79606500 -0.60364000

H -4.79161800 -1.99186100 - 1.26387200

O $-5.39537400-0.488016001 .44806900$

H -6.26022900 -0.83576000 1.21297300

O $-4.712730000 .77463000-0.87302300$

H -5.36791800 $1.42110700-0.59341100$ TS2

Si -0.898852000 .324530001 .24794600$ O - 0.827428001 .800064000 .45511300

O $-0.25614000-0.62380100-0.01381700$

Si $0.05388100-1.85253700-1.01644400$

O $1.57729500-2.41913100-0.72856200$

Si $2.68597800-1.750102000 .28100100$
O $3.01616200-0.22835900-0.25196000$

Si $2.651761001 .35433900-0.05107500$

O $1.424533001 .77102400-1.05819900$

Si -0.16706100 2.15562800-0.99946900

O $2.21179300-1.749387001 .84359200$

O $4.01721400-2.698526000 .11211500$

H $4.77795900-2.477998000 .65645100$

O $3.917355002 .27937100-0.53993700$

O 2.304044001 .681088001 .51590900

O $-0.366327003 .76138400-1.30608500$

H $0.079885004 .37440100-0.71473700$

O -0.93485300 $1.35925100-2.22728900$

H - $1.841642001 .08766400-1.98942500$

O 0.270798000 .012832002 .38692100

O -2.43418200 -0.33980900 1.25937100

O -0.93279600 -3.14675000-0.79053100

H - $1.87124500-2.89607200-0.72349900$

O -0.03711900 -1.30090400 -2.56732300

H - $0.25171800-0.35189500-2.61343700$

H $1.46361200-1.150585002 .09551900$

H 1.614226001 .114706001 .92876600

H $4.773528002 .08814700-0.14691300$

O -1.44314900 1.361679002 .95075400

H -0.394944000 .757896003 .06817200$

H -1.30027900 2.307804002.84097500

Si -3.60170500 -0.46699900 0.14362600

O -3.45180200 $0.61897700-1.10448100$

O $-3.45819000-1.97443500-0.52858900$

O $-5.08691400-0.199793000 .79199800$

H -3.89787800 $1.46287800-0.97216200$

H -4.12064600 -2.21999800 -1.18291100

H -5.24923700 -0.52316700 1.68303200

Product

Si -0.888618000 .526110000 .80990100$

O -0.48817100 2.05009600 0.26721500

O $-0.64522500-0.44194000-0.49250100$

Si -0.24662100 - $1.51302000-1.64283400$

O $1.00627200-2.43281000-1.10033300$

Si $2.03514700-2.192928000 .16630900$

O $2.81979700-0.77089900-0.05470600$

Si 2.925075000 .804236000 .38667600

O $2.016937001 .68223900-0.66342600$

Si $0.611270002 .48385900-0.86563900$

O $1.25164700-2.231273001 .59835500$ 
O $3.19154600-3.359312000 .11890600$ H $2.89420900-4.267634000 .01744000$ O 4.460381001 .340385000 .16708000 O 2.445337001 .032154001 .92956900 O $0.795025004 .11392400-0.83464500$ H 0.785980004 .538645000 .02818300 O $0.161619002 .07351600-2.40597000$ H -0.62474400 2.50332500 -2.75740300 O -0.01930500 0.07208000 2.06220400 O -2.52050200 0.526964001 .08283400 O $-1.52269200-2.49234500-1.96181900$ H -2.31618400 -2.26893000 -1.44268700 O $0.22046400-0.71126500-2.99702100$ H $0.236700000 .25779700-2.89917100$ H $0.71799300-1.422521001 .82505700$ H 1.515547000 .715885002 .10305100 H 5.163495000 .845552000 .59683900 O -0.93089400 -0.09164600 4.59798000 H -0.61151100 -0.01639800 3.66816100 H -0.930919000 .806321004 .94037800$ Si -3.77227100 -0.01221600 0.18324400 O -3.76793600 $0.86632600-1.20462000$ O -3.63364900 - $1.60499700-0.26081200$ O -5.15953300 0.089358001 .05788400 H -4.43958300 $0.66266400-1.86202500$ H -3.84366000 -2.26072000 0.41221300 H -5.29199400 0.877018001 .59337100 